\title{
Thermal parameter identification for non-Fourier heat transfer from molecular dynamics
}

\author{
Amit Singh, Ellad B. Tadmor* \\ Department of Aerospace Engineering and Mechanics, The University of Minnesota, \\ Minneapolis, MN 55455
}

\begin{abstract}
Fourier's law leads to a diffusive model of heat transfer in which a thermal signal propagates infinitely fast and the only material parameter is the thermal conductivity. In micro- and nano-scale systems, non-Fourier effects involving coupled diffusion and wavelike propagation of heat can become important. An extension of Fourier's law to account for such effects leads to a Jeffreys-type model for heat transfer with two relaxation times. We propose a new Thermal Parameter Identification (TPI) method for obtaining the Jeffreys-type thermal parameters from molecular dynamics simulations. The TPI method makes use of a nonlinear regression-based approach for obtaining the coefficients in analytical expressions for cosine and sine-weighted averages of temperature and heat flux over the length of the system. The method is applied to argon nanobeams over a range of temperature and system sizes. The results for thermal conductivity are found to be in good agreement with standard Green-Kubo and direct method calculations. The TPI method is more efficient for systems with high diffusivity and has the advantage, that unlike the direct method, it is free from the influence of thermostats. In addition, the method provides the thermal relaxation times for argon. Using the determined parameters, the Jeffreys-type model is able to reproduce the molecular dynamics results for a short-duration heat pulse where wavelike propagation of heat is observed thereby confirming the existence of second sound in argon. An implementation of the TPI method in MATLAB is available as part of the online supplementary material.
\end{abstract}

\footnotetext{
${ }^{*}$ Corresponding author

Email address: tadmor@umn.edu (Ellad B. Tadmor)
}

Preprint submitted to Journal of Computational Physics

June 26, 2015

(C) 2015. This manuscript version is made available under the Elsevier user license http://www.elsevier.com/open-access/userlicense/1.0/ 
Keywords:

Molecular dynamics, Non-Fourier heat transfer, Jeffreys-type model, Cattaneo model, Transient heat transfer

\section{Introduction}

Understanding heat transfer at the molecular level is of both academic and technological interest. In any theory describing heat transfer, a constitutive assumption is necessary to relate the heat flux vector $\boldsymbol{q}(\boldsymbol{x}, t)$ and the temperature gradient $\boldsymbol{\nabla} \theta(\boldsymbol{x}, t)$, where $\boldsymbol{x}$ is a position in the current configuration and $t$ is the time. Such a relation can be very general as long as it satisfies constraints on its form imposed by the second law of thermodynamics (see for example Section 6.2.2 in Ref. 1]). The simplest theory of heat transfer adopts Fourier's law of heat conduction in which a linear relation is assumed between the heat flux and temperature gradient for a homogeneous isotropic body,

$$
\boldsymbol{q}(\boldsymbol{x}, t)=-k \boldsymbol{\nabla} \theta(\boldsymbol{x}, t),
$$

where $k$ is the thermal conductivity of the material. As we review in Section 2, Fourier's law leads to the heat equation, a parabolic partial differential equation (PDE) that describes heat transfer as a diffusive process. The heat equation faces the conceptual problem that a sudden change of temperature at any point in space is felt instantly everywhere. It can be said that diffusion assumes an infinite speed of propagation (see p. 42 in Ref. [2]). In reality, a finite time is involved in heat transfer in solids, which occurs through collisions between energy carriers - free electrons and phonons - and also by scattering of these carriers at boundaries and material defects. Phonons are the major heat carriers for insulators and semiconductors, whereas free electrons are the dominant carriers in metals [3]. The finite speed of heat transfer was recognized by many including Chester [4], and Joseph and Preziosi [2] in their seminal paper on "heat waves." In the latter work as well as in earlier contributions by Cattaneo [5, 6] and Vernotte [7, 8], non-Fourier heat transfer models were proposed in which the relationship between $\boldsymbol{q}(\boldsymbol{x}, t)$ and $\boldsymbol{\nabla} \theta(\boldsymbol{x}, t)$ is more complex. The Cattaneo-Vernotte (CV) model leads to a hyperbolic PDE which combines wave-like and diffusive features. This model predicts that thermal discontinuities propagate at a finite velocity $c$. A more general Jeffreys-type model leads to a PDE in which thermal discontinuities are smoothed out as they propagate. The Jeffreys-type model includes in 
addition to the thermal conductivity $k$, two relaxation times $\tau_{q}$ and $\tau_{\theta}$. The $\mathrm{CV}$ and Fourier models can be obtained as special cases of the Jeffreys-type model. Various other heat-propagation models have been proposed and are comprehensively reviewed by Straughan [9].

While the question whether non-Fourier effects are present at the macroscale is controversial (see for example Refs. [10-13]), there is no doubt that such effects exist and play an important role in micro and nano-scale systems [14]. This has great technological importance since modern microfabrication processes involve the miniaturization of devices that contain semiconductors, insulators and metallic layers on micro and nanometer scales. Fourier heat transfer breaks down when the characteristic lengths associated with the device approach the mean-free path of the energy carriers (electrons and phonons) [15]. For example, Fourier's law cannot explain the thickness dependence of the thermal conductivity of silicon [15 17]. Pilgrim et al. [18] studied the transient behavior of heat pulses in samples of GaAs and found that while the Fourier model successfully captured the thermal effects in larger samples, the CV model was more suitable for samples of smaller size. Lor and Chu [19] found that the CV model explained the heat transport in a thin film of a high-temperature yttrium barium copper oxide superconductor deposited on various substrates. Wagner et al. [20] developed an energy-conserving method to couple atomistic MD regions to continuum finite element regions for nanoscale thermal problems, and suggested using a CV model to describe heat transfer in continuum regions.

Non-Fourier effects are also important on very short timescales. For example, when laser pulses are used for annealing semi-conductors, oscillatory heat flux boundary conditions (BCs) must be considered [21, 22] that cannot be described by Fourier theory. Similarly, thermal resonance phenomena due to the excitation of a body heat source oscillating at a given frequency cannot be explained by Fourier's Law [23 26]. Just as displacement waves due to mechanical vibrations can lead to resonance in the displacement amplitude, a resonance phenomenon may occur in the wave amplitude of the temperature when a material is subjected to oscillatory heat flux BCs. Research in non-Fourier heat conduction also finds application in cryogenic systems, spacecraft thermal control coatings and superconducting thin films [27], reactor fuel rods [28], phase changes [29, 30], planetary heat propagation [31, 32], chemotaxis [33], skin burns [34], and other applications [9, 35, 36].

Heat transfer in atomic-scale systems has been explored computationally with molecular dynamics (MD) using two primary approaches: (1) the 
Green-Kubo relations within equilibrium MD; and (2) direct measurement of the thermal response in a nonequilibrium MD (NEMD) simulation. The Green-Kubo relations, which are based on the fluctuation-dissipation theorem, relate thermal conductivity to an integral of the time correlation function of the heat current (see Eq. (14) below). In the direct method, a thermal gradient is imposed on the system by thermostatting its ends and measuring the heat flux at steady state from which the thermal conductivity can be readily computed using Eq. (1). Ladd et al. [37] carefully compared the formally exact Green-Kubo relations with the direct NEMD approach and a method based on phonon perturbation theory for a face-centered cubic (fcc) lattice with "soft-sphere" interactions. They found that the Green-Kubo method works well over a broad range of temperatures but requires long simulation times to obtain good statistics. The other two methods are more efficient but were found to have a limited range of applicability. In particular, NEMD works well at higher temperatures whereas phonon perturbation theory is appropriate only at low temperatures. More recently, Schelling et al. [38] confirmed these results and showed that at high temperature the direct NEMD method and the Green-Kubo approach give similar values for the thermal conductivity of silicon.

The MD studies described above have been primarily focused on Fourier heat transfer. However, Non-Fourier effects have also been observed in atomistic simulations. An important early study of this kind was performed by Tsai and MacDonald [39] who studied the response of a body-centered cubic (bcc) lattice to a strong heat pulse applied at the boundary. The authors observed that the disturbance propagated into the bulk "as a combination of first and second sound superimposed on a diffusive background." Here "first sound" refers to the propagation of a stress wave and "second sound" to the propagation of a thermal wave (see for example Ref. (4)). The "diffusive background" is captured by Fourier's model. No attempt was made to relate these results to non-Fourier heat transfer models. Later, Volz [40] performed similar NEMD simulations on a solid beam of fcc argon and tested the validity of the CV model. It was found that the CV model is adequate under equilibrium conditions, but does a poor job of describing the microscopic response in the initial nonequilibrium regime after the injection of heat. More recently, Mohamed and Srivastava [41] found that heat pulses of picosecond duration in single-walled carbon nanotubes induce several wave packets that travel at different propagation speeds. The largest amount of energy was found in the waves moving at the second sound speed. Single-walled nan- 
otubes were also studied by Shiomi and Maruyama [42] who concluded that heat is conducted in a wavelike fashion. They also found that the flux profiles cannot be predicted by the hyperbolic wave equation (the CV model), but can be captured by taking the dual relaxation time scale into account, thus confirming the validity of Jeffreys-type models. Liu et al. [43] studied transient thermal conduction in pure and vacancy-containing argon films by applying a temperature jump to one end of the film and then calculating the thermal wave speed and relaxation times appearing in the non-Fourier models. They found that the wave speed is the same in both pure argon films and argon films with vacancy defects.

A practical problem with the NEMD approach is the existence of discontinuous jumps in temperature at the model boundaries where temperature is controlled. These jumps, which are referred to as the Kapitza effect, are due to phonon scattering at the interface between thermostatted and unthermostatted regions that causes a sharp change in thermal conductivity at the interface. The scattering occurs because thermostatting algorithms corrupt the dynamics of the thermostatted atoms [44]. It is important to stress that this is an artificial Kapitza effect due to the presence of the thermostat. Real physical temperature jumps can occur at the interface between two different materials, but this is not the case here. The artificial Kapitza effect leads to a discontinuity in the temperature gradient at the interface, giving rise to a jump in the temperature. Tenenbaum et al. [45] observed this phenomena in their early simulations and attempted to quantify the jumps. Aoki and Kusnezov [46] developed a quantitative description of the jumps by studying nonequilibrium properties of a one-dimensional (1D) lattice Hamiltonian (see the book by Pitaveskii and Lifshitz [47] for more details on their approach). Other researchers have also studied this effect or observed similar temperature jumps in their simulations [38, 48 51]. (We discuss a phenomenological model for addressing the artificial Kapitza effect in MD simulations in a separate publication [52].)

The discussion above makes it clear that there is interest in determining the non-Fourier thermal behavior of material systems especially at the microand nano-scales where MD can be applied. The Green-Kubo and direct NEMD methods can be used to obtain the thermal conductivity $k$ of the material but can be slow and difficult to apply accurately and they do not provide the thermal relaxation times.

In this work, we extend the approach of Daly et al. [53] for computing thermal conductivity by monitoring the decay of an initially imposed 
sinusoidal temperature distribution, to extract the Jeffreys-type model parameters, $k, \tau_{q}$ and $\tau_{\theta}$, from NEMD simulations of a $1 \mathrm{D}$ nanobeam. The nanobeam is first equilibrated with a sinusoidal temperature distribution using independent thermostats at equidistant positions along the length of the beam. The thermostats are then removed and the nanobeam evolves under under constant energy $(N V E)$ conditions. During this nonequilibrium unsteady process, time-averaged temperature and heat-flux profiles along the nanobeam are computed and stored at regular intervals. These profiles are fitted to analytical solutions of the CV and Jeffreys-type PDEs. Thus a nonlinear regression-based parameter identification procedure is applied in which the coefficients of the governing PDE are obtained by analyzing the NEMD data. We refer to this method for obtaining thermal parameters as "TPI" (thermal parameter identification). The advantage of this approach is that relatively few profiles are needed to obtain the parameters to good accuracy which means that the NEMD simulation only needs to be run for a short time following the initial sinusoidal temperature equilibration.

The structure of the paper is as follows. In Section 2, we begin with a brief overview of Fourier and non-Fourier heat transfer models. In Section 3 , we describe the NEMD simulations, the details of the binning procedure used to obtain temperature profiles, and the thermal conductivity and TPI calculations. In Section 4, we present the TPI results for argon nanobeams with Lennard-Jones (LJ) pair potential interactions. The results for the bulk thermal conductivity are found to be in good agreement with those obtained using standard Green-Kubo and direct NEMD calculations. In addition, TPI obtains the relaxation times $\tau_{q}$ and $\tau_{\theta}$. It is found that a Jeffrey-type model with TPI parameters is able to describe the observed thermal response, whereas a simple Fourier model is inadequate. It is also observed that $\tau_{\theta}$ is very small in comparison with $\tau_{q}$. In Section 5, we study the spreading of an isolated heat pulse applied to an argon nanobeam. It is found that the resulting temperature profiles are in good agreement with those obtained from a numerical solution of the Jeffreys-type model using the Jeffreys parameters obtained by fitting to a different thermal boundary-value problem. In addition, the speed of heat wave propagation is computed from the atomistic data and found to agree with the predictions of the continuum Jeffreys-type model. We conclude in Section 6 with a summary. 


\section{Fourier and non-Fourier heat transfer models}

\subsection{Fourier's model}

Fourier's law of heat conduction, described in Eq. (11), relates the heat flux vector and the temperature gradient. For a rigid solid body without heat sources, the energy equation is (see for example Section 10.2 in Ref. [1] )

$$
\frac{\partial e}{\partial t}=-\nabla \cdot \boldsymbol{q}
$$

where $e=e(\boldsymbol{x}, t)$ is the internal energy per unit volume. From the first law of thermodynamics, for rigid solid bodies where mechanical work cannot change the volume or shape of the system, we have

$$
\frac{\partial e}{\partial \theta}=\gamma
$$

where $\gamma=\rho c_{v}$ is volumetric heat capacity, which is a product of the mass

density and the specific heat at constant volume. Using Eqs. (1)-(3), we obtain Fourier's heat equation:

$$
\frac{\partial \theta}{\partial t}=\frac{k}{\gamma} \nabla^{2} \theta
$$

This is a parabolic PDE which describes heat transfer as a diffusive process.

\subsection{Non-Fourier models}

Various models have been proposed in the literature for understanding heat conduction at finite wave speeds [2, 36]. Collectively, such models are referred to as non-Fourier heat conduction models. The basic idea is to introduce a richer physics into the heat flux constitutive relation in Eq. (1). Cattaneo [5, [6] and Vernotte [7, 8] proposed the following model for heat flux:

$$
\tau_{q} \frac{\partial \boldsymbol{q}}{\partial t}(\boldsymbol{x}, t)+\boldsymbol{q}(\boldsymbol{x}, t)=-k \boldsymbol{\nabla} \theta(\boldsymbol{x}, t) .
$$

This relation accounts for an increase in the heat flux vector due to phonon collisions over a relaxation time $\tau_{q}$. Applying to Eq. (5), the same procedure that leads from Eq. (11) to the heat equation in Eq. (41), yields the CV wave equation,

$$
\frac{\partial^{2} \theta}{\partial t^{2}}+\frac{1}{\tau_{q}} \frac{\partial \theta}{\partial t}=c^{2} \nabla^{2} \theta
$$


This is a hyperbolic PDE in which thermal waves travel at a finite wave speed,

$$
c=\sqrt{k / \gamma \tau_{q}}
$$

and are attenuated by diffusive effects over time. This model can describe the propagation of temperature discontinuities and hence thermal shocks. The model is also known as Telegraph equation and first attracted serious attention when treated by Poincaré [54] in 1893, who analyzed the equation with some generality using Fourier transformations.

In an alternative approach, Joseph and Preziosi [2] based on earlier work by Gurtin and Pipkin [55], and Nunziato [56], introduced the concepts of effective thermal conductivity and effective heat capacity to take into account non-Fourier behavior. They obtained an integro-differential equation for the heat flux vector,

$$
\boldsymbol{q}(\boldsymbol{x}, t)=-k_{1} \boldsymbol{\nabla} \theta(\boldsymbol{x}, t)-\frac{k_{2}}{\tau_{q}} \int_{-\infty}^{t} \boldsymbol{\nabla} \theta\left(\boldsymbol{x}, t^{\prime}\right) e^{-\left(t-t^{\prime}\right) / \tau_{q}} d t^{\prime},
$$

where $k_{1}$ and $k_{2}$ are the effective conductivity and the elastic conductivity, respectively. The motivation came from a model developed by Jeffreys [57] for a viscoelastic liquid, where shear stress and shear strain were related in a manner analogous to heat flux and temperature gradient in Eq. (8). This was a special model obtained after generalization of Boltzmann's equation of linear viscoelasticity [58], and was inspired by the work done by Maxwell [59] and Boltzmann [60].

Differentiating Eq. (8) with respect to $t$ and using the Leibniz integral rule, a heat-flux equation of the Jeffreys-type is obtained:

$$
\tau_{q} \frac{\partial \boldsymbol{q}}{\partial t}(\boldsymbol{x}, t)+\boldsymbol{q}(\boldsymbol{x}, t)=-k \boldsymbol{\nabla} \theta(\boldsymbol{x}, t)-\tau_{q} k_{1} \frac{\partial \boldsymbol{\nabla} \theta}{\partial t}(\boldsymbol{x}, t),
$$

where $k=k_{1}+k_{2}$. When $k_{1}=0$, Eq. (9) reduces to Cattaneo's law in Eq. (5). Following Tzou's approach [61, 62], $k_{1}$ can be written in terms of a new relaxation time $\tau_{\theta}$ as $k_{1}=k \tau_{\theta} / \tau_{q}$, leading to the following heat-flux equation:

$$
\tau_{q} \frac{\partial \boldsymbol{q}}{\partial t}(\boldsymbol{x}, t)+\boldsymbol{q}(\boldsymbol{x}, t)=-k \boldsymbol{\nabla} \theta(\boldsymbol{x}, t)-k \tau_{\theta} \frac{\partial \boldsymbol{\nabla} \theta}{\partial t}(\boldsymbol{x}, t) .
$$






Figure 1: Schematic of a slender beam.

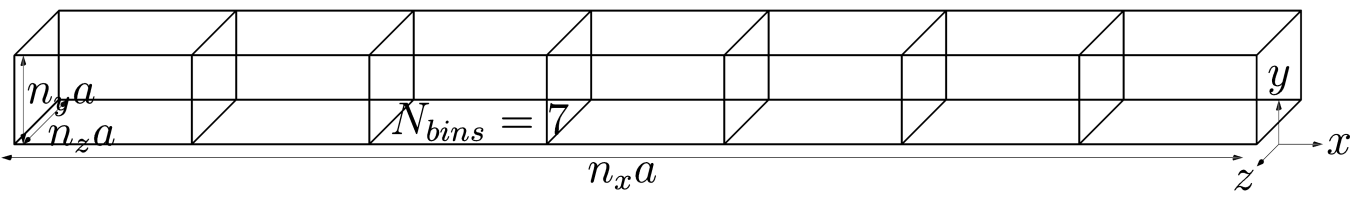

Figure 2: Division of the nanobeam into bins.

The procedure outlined in Section 2.1 then leads to a Jeffreys-type equation for the temperature:

$$
\frac{\partial^{2} \theta}{\partial t^{2}}(\boldsymbol{x}, t)+\frac{1}{\tau_{q}} \frac{\partial \theta}{\partial t}(\boldsymbol{x}, t)=c^{2} \nabla^{2} \theta(\boldsymbol{x}, t)+\frac{k_{1}}{\gamma} \nabla^{2} \frac{\partial \theta}{\partial t}(\boldsymbol{x}, t) .
$$

This is a parabolic PDE (when $k_{1} \neq 0$ ) in which, similar to the CV model, thermal disturbances propagate at the wave speed $c$. However, in contrast to $\mathrm{CV}$, thermal shock fronts are rounded and smoothed out over time. (See Section III in Ref. [2] for an example showing the differences between the CV and Jeffreys-type models.)

\section{Obtaining thermal parameters using molecular dynamics}

Our objective is to obtain the thermal parameters appearing in the continuum models described in Section 2 by performing MD simulations. As the test system, we choose a slender nanobeam of length $L$ and square crosssection of side $W$ with $L \gg W$ (see Fig. 1). The thermal conductivity is obtained using the standard Green-Kubo and NEMD direct methods outlined in Section 3.2, and all three Jeffreys-type model parameters are obtained using the new regression-based TPI approach proposed in Section 3.3. We begin by describing a binning procedure for MD simulations, which is required for both the NEMD direct method and the TPI approach. 


\subsection{Binning for spatial and temporal averaging}

The integration of the equations of motion by MD provides the positions and momenta of all particles in the system as a function of time. We are interested in computing the time-dependent profiles along the beam of thermodynamic properties, such as the temperature $\theta(x, t)$ and heat flux $\boldsymbol{q}(x, t)$. Such profiles are needed for computing thermal properties and also for the imposition of thermal BCs. In order to do so, we divide the beam into $N_{\text {bins }}$ bins of equal size as shown for example in Fig. 2 for $N_{\text {bins }}=7$. The value of a thermodynamic property is then obtained as a spatial average over an appropriate phase function across all atoms in a bin. For example the instantaneous temperature of bin $n$ is

$$
\theta_{n}^{\text {inst }}=\frac{2 \mathcal{K}}{3 N_{n} k_{\mathrm{B}}}
$$

where $\mathcal{K}$ is kinetic energy (with rigid-motion removed [63]) of all $N_{n}$ atoms in bin $n$ and $k_{\mathrm{B}}$ is Boltzmann's constant. The temperature follows as the phase average of the instantaneous temperature, which in an MD simulation is obtained by performing a time average:

$$
\theta_{n}(t)=\left\langle\theta_{n}^{\text {inst }}\right\rangle=\frac{1}{\Delta_{t}} \int_{t}^{t+\Delta_{t}} \theta_{n}^{\text {inst }}(\tau) d \tau .
$$

In this relation $\Delta_{t}$ is a time interval which must be taken sufficiently long to ensure that local thermodynamic equilibrium (LTE) is established but not so long as to smear out nonequilibrium effects. The basic assumption in LTE is that during a nonequilibrium process, there exists a timescale over which thermodynamic variables, such as temperature, are well-defined and obey standard thermodynamic relations, such as equations of state [64]. In order to improve the statistics, and reduce the necessary $\Delta_{t}$, simulations can be repeated multiple times. Bin averages are then computed over all realizations of a given bin. This reduces the fluctuation $\delta \theta_{n}$ and therefore allows for a smaller averaging time interval. In most of our simulations we observe that LTE assumption is correct for $\Delta_{t} \geq 0.2 \mathrm{ps}$ when bin averages are computed over more than five realizations [39, 40].

\subsection{Standard methods for calculating thermal properties}

The non-Fourier Jeffreys-type model described in Section 2.2 includes three material parameters: the steady-state thermal conductivity $k$ and two 
relaxation times $\tau_{q}$ and $\tau_{\theta}$. Two standard approaches for calculating $k$ are (1) the Green-Kubo method using equilibrium MD, and (2) the "direct method" using NEMD. These approaches are described below, followed in Section 3.3 by the TPI method which obtains all three non-Fourier parameters.

\subsubsection{Green-Kubo method}

The Green-Kubo method is based on the fluctuation-dissipation theorem. The thermal conductivity is 1

$$
\boldsymbol{k}=\frac{1}{V k_{\mathrm{B}} \theta^{2}} \int_{0}^{\infty}\langle\boldsymbol{J}(t) \otimes \boldsymbol{J}(0)\rangle_{\nabla_{\theta=0}} d t,
$$

where $V$ is the volume and $\boldsymbol{J}=V \boldsymbol{q}$ is the microscopic heat current. For practical calculations, the thermal conductivity tensor in Eq. (14) can also be written as [38]

$$
\begin{aligned}
\boldsymbol{k} & =\lim _{\tau_{I} \rightarrow \infty} \frac{1}{V k_{\mathrm{B}} \theta^{2}} \int_{0}^{\tau_{I}}\langle\boldsymbol{J}(t) \otimes \boldsymbol{J}(0)\rangle_{\nabla_{\theta=0}} d t \\
& =\lim _{\substack{S, I \rightarrow \infty \\
S \gg I}} \frac{\Delta t_{\mathrm{MD}}}{V k_{\mathrm{B}} \theta^{2}} \sum_{a=1}^{I} \frac{1}{S-a} \sum_{b=1}^{S-a} \boldsymbol{J}_{a+b} \otimes \boldsymbol{J}_{b},
\end{aligned}
$$

where $\Delta t_{\mathrm{MD}}$ is the MD time step, $S$ is the total number of simulation steps, $I$ is the total number of integration steps, $\tau_{I}$ is the integration time $\left(\tau_{I}=\right.$ $\left.I \Delta t_{\mathrm{MD}}\right)$, and $\boldsymbol{J}_{m}=\boldsymbol{J}\left(m \Delta t_{\mathrm{MD}}\right)$. In order to obtain a bulk property, $\tau_{I}$ must be very large, i.e., $I$ must be a large number.

We consider a system of $N$ atoms with mass $m_{i}$ and position $\boldsymbol{r}_{i}$. The distance between atoms $i$ and $j$ is $r_{i j}=\left\|\boldsymbol{r}_{j}-\boldsymbol{r}_{i}\right\|$. The velocity of atom $i$ relative to the center-of-mass velocity of the nanobeam (or bin) is denoted $\tilde{\boldsymbol{v}}_{i}$. For pair potential interactions, the heat current $\boldsymbol{J}$ is [37, 66 68],

$$
\boldsymbol{J}=\sum_{i=1}^{N} e_{i} \tilde{\boldsymbol{v}}_{i}+\sum_{\substack{i, j \\ i<j}}^{N}\left[\boldsymbol{f}_{i j} \cdot \frac{\tilde{\boldsymbol{v}}_{i}+\tilde{\boldsymbol{v}}_{j}}{2}\right]\left(\boldsymbol{r}_{i}-\boldsymbol{r}_{j}\right)
$$

\footnotetext{
${ }^{1}$ This expression is obtained by writing the energy equation in terms of fluctuations around the equilibrium value in the linear regime and the wave-vector-dependent internal energy density autocorrelation function. A more detailed analysis is available in Ref. [65].
} 


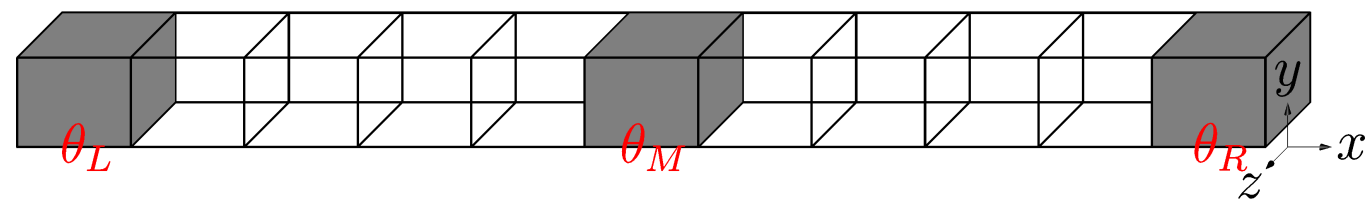

Figure 3: Schematic diagram of a beam partitioned into bins with periodic BCs. Shaded regions are thermostatted.

Here $e_{i}$ is the local site energy of atom $i$ which includes both kinetic and potential terms. For a monoatomic system with pair potential interactions this is 2

$$
e_{i}=\frac{1}{2} m_{i} \tilde{\boldsymbol{v}}_{i}^{2}+\frac{1}{2} \sum_{\substack{j \\ j \neq i}}^{N} \phi\left(r_{i j}\right)
$$

where $\phi(r)$ is the pair potential function. Also appearing in Eq. (16), $\boldsymbol{f}_{i j}$ is the force on atom $i$ due to the presence of atom $j$. For a pair potential this is

$$
\boldsymbol{f}_{i j}=\phi^{\prime}\left(r_{i j}\right) \frac{\boldsymbol{r}_{j}-\boldsymbol{r}_{i}}{r_{i j}}
$$

\subsubsection{Direct method}

In the direct method, a temperature gradient is created in the nanobeam by thermostatting the left and right ends of the nanobeam at the same temperature, i.e. $\theta_{\mathrm{L}}=\theta_{\mathrm{R}}$, and maintaining the middle bin at a temperature $\theta_{\mathrm{M}}$ (see Fig. 3i) with periodic BCs applied in all directions. Thus for a nanobeam of length $L$, the $\mathrm{BCs}$ are

$$
\theta(0, t)=\theta_{\mathrm{L}}, \quad \theta(L, t)=\theta_{\mathrm{R}}, \quad \theta(L / 2, t)=\theta_{\mathrm{M}} .
$$

The temperature gradients to the left and right of the middle bin are calculated by fitting straight lines to the steady-state temperature profiles in the regions away from thermostats. For this purpose, the two nearest bins

\footnotetext{
${ }^{2}$ Note that the concept of a "site energy" is ill-defined for more complex atomic interactions and even for pair potentials in systems containing more than one atom type. See Ref. [68] for more details on this.
} 
to each thermostatted bin are excluded from the linear fitting. Then the thermal conductivity along the $x$ direction is estimated from Eq. (11) as

$$
k=\frac{1}{2}\left(\langle q\rangle_{\mathrm{L}}\left(\frac{\Delta \theta}{\Delta x}\right)_{\mathrm{L}}^{-1}+\langle q\rangle_{\mathrm{R}}\left(\frac{\Delta \theta}{\Delta x}\right)_{\mathrm{R}}^{-1}\right)
$$

where the $x$-component of the heat flux vector is obtained from Eq. (16) $(q=$ $J_{x} / V_{\text {bin }}$, where $V_{\text {bin }}$ is the volume of each bin) and $\Delta \theta / \Delta x$ is the temperature gradient obtained from the linear fit. The subscripts $\mathrm{L}$ and $\mathrm{R}$ refer to left and right sides of the nanobeam, and $\langle q\rangle$ represents the average of the $x$ component of the heat flux vector of all bins included in the linear fitting.

The thermal conductivity depends on the length (size) of the beam for two reasons [38, 69, 70]: (a) shorter nanobeams have fewer phonon modes available and therefore include less phonon-phonon scatterings; (b) when the nanobeam length is smaller than the bulk phonon mean-free paths, phononboundaries scattering effects increase significantly.

\subsection{Regression-based thermal parameter identification}

In the TPI approach, an NEMD simulation is performed starting with initial conditions similar to Daly et al. [53], in which the nanobeam has a sinusoidal temperature distribution of the form:

$$
\theta(x, 0)=\theta_{0}+\Delta \theta_{0} \cos (2 \pi x / L), \quad 0 \leq x \leq L,
$$

where $\theta_{0}$ and $\Delta \theta_{0}$ are the initial temperature and fluctuation amplitude. The initial temperature profile is applied by dividing the nanobeam into bins (as shown in Fig. 21) and individually thermostatting each bin in accordance with Eq. (21). The thermostats are then switched off and the nanobeam is left to evolve freely subject to periodic BCs, $\theta(0, t)=\theta(L, t)$, for $t \geq 0$. The relaxation of the temperature and heat flux profiles are analyzed to calculate the thermal parameters by comparing to the following analytical solution for the non-Fourier models.

Given the slender nanobeam geometry, we approximate the three-dimensional heat transfer problem as 1D. Using Eq. (7), we rewrite Eq. (11) in 1D as

$$
\tau_{q} \frac{\partial^{2} \theta}{\partial t^{2}}+\frac{\partial \theta}{\partial t}=\frac{k}{\gamma} \frac{\partial^{2} \theta}{\partial x^{2}}+\frac{k}{\gamma} \tau_{\theta} \frac{\partial^{3} \theta}{\partial x^{2} \partial t}
$$


Since there are no heat sources in the system, it is reasonable to assume that the temperature rate of change at time $t=0$ is zero, i.e.,

$$
\frac{\partial \theta}{\partial t}(x, 0)=0, \quad 0 \leq x \leq L
$$

Rather than solving for $\theta:(0, L) \times(0,+\infty) \rightarrow \mathbb{R}_{+}$, where $\mathbb{R}_{+}$is the set of non-negative real numbers, we solve for the perturbation $\phi(x, t)$ in the temperature from the mean temperature $\theta_{0}$, defined as $\phi(x, t):=\theta(x, t)-\theta_{0}$. We thus seek to solve,

$$
\begin{cases}\tau_{q} \frac{\partial^{2} \phi}{\partial t^{2}}+\frac{\partial \phi}{\partial t}=\alpha \frac{\partial^{2} \phi}{\partial x^{2}}+\alpha \tau_{\theta} \frac{\partial^{3} \phi}{\partial x^{2} \partial t} & , \\ \phi(0, t)=\phi(L, t), & t \geq 0, \\ \phi(x, 0)=\Delta \theta_{0} \cos (2 \pi x / L), & 0 \leq x \leq L, \\ \frac{\partial \phi}{\partial t}(x, 0)=0, & 0 \leq x \leq L .\end{cases}
$$

where $\alpha=k / \gamma$ is thermal diffusivity. Using the method of separation of variable, we can write all solutions of the above problem in the special form $\phi(x, t)=X(x) T(t)$, where $X(x)$ and $T(t)$ are unknown functions of $x$ and $t$. Substituting this into Eq. (24), we obtain

$$
\begin{array}{r}
{\left[\tau_{q} \ddot{T}(t)+\dot{T}(t)\right] X(x)=\alpha X^{\prime \prime}(x)\left[T(t)+\tau_{\theta} \dot{T}(t)\right]} \\
\Longrightarrow \frac{X^{\prime \prime}(x)}{X(x)}=\frac{1}{\alpha} \frac{\tau_{q} \ddot{T}(t)+\dot{T}(t)}{T(t)+\tau_{\theta} \dot{T}(t)}=-\omega^{2}
\end{array}
$$

where $\omega$ is the separation constant independent of $x$ and $t$. Using the initial and boundary conditions in Eq. (24), Eq. (25) leads to two separate problems:

$$
\left\{\begin{array}{l}
X^{\prime \prime}(x)+\omega^{2} X(x)=0 \\
X(0)=X(L), X(x)=\Delta \theta_{0} \cos (2 \pi x / L),
\end{array}\right.
$$

and

$$
\left\{\begin{array}{l}
\tau_{q} \ddot{T}(t)+\left(1+\omega^{2} \alpha \tau_{\theta}\right) \dot{T}(t)+\omega^{2} \alpha T(t)=0, \\
T(0)=1, \dot{T}(0)=0 .
\end{array}\right.
$$

The solution to Eq. (26) is $X(x)=\Delta \theta_{0} \cos (\omega x)$, with $\omega=2 \pi / L$. The 
solution to Eq. (27) is

$$
T\left(t ; \lambda_{i}\right)= \begin{cases}e^{\lambda_{1} t}\left(\cos \lambda_{2} t-\frac{\lambda_{1}}{\lambda_{2}} \sin \lambda_{2} t\right), & \text { if } \lambda_{2}^{2}>0, \\ \frac{e^{\lambda_{1}} t}{2 \lambda_{3}}\left[\left(\lambda_{3}-\lambda_{1}\right) e^{\lambda_{3} t}+\right. & \\ \left.\left(\lambda_{3}+\lambda_{1}\right) e^{-\lambda_{3} t}\right], & \text { if } \lambda_{2}^{2}<0, \\ e^{\lambda_{1} t}\left(1-\lambda_{1} t\right), & \text { if } \lambda_{2}^{2}=0,\end{cases}
$$

where $\lambda_{i} ; i=1,2,3$, are parameters defined by

$$
\lambda_{1}=-\frac{1}{2 \tau_{q}}\left(1+\omega^{2} \alpha \tau_{\theta}\right), \lambda_{2}=\sqrt{\omega^{2} \frac{\alpha}{\tau_{q}}-\lambda_{1}^{2}}, \lambda_{3}^{2}=-\lambda_{2}^{2} .
$$

Thus the solution to the Jeffreys-type model in Eq. (22) subject to the initial conditions in Eqs. (21) and (23) under periodic BCs is given by

$$
\theta(x, t)=\theta_{0}+\Delta \theta_{0} \cos (2 \pi x / L) T(t), \quad 0 \leq x \leq L,
$$

where $T(t)$ is given by Eq. (28). The existence, uniqueness and stability of the solution in Eq. (30) is demonstrated in the Appendix.

We will also require an expression for the $x$-component of the heat flux vector. We start with a $1 \mathrm{D}$ version of Eq. (8) :

$$
q(x, t)=-k_{1} \frac{\partial \theta}{\partial x}(x, t)-\frac{k_{2}}{\tau_{q}} \int_{0}^{t} \frac{\partial \theta}{\partial x}\left(x, t^{\prime}\right) e^{-\left(t-t^{\prime}\right) / \tau_{q}} d t^{\prime},
$$

where the lower limit in the integral is $t^{\prime}=0$, since the initial conditions are known at this time 3 Substituting Eq. (30) into Eq. (31), we obtain

$$
q(x, t)=\pi \Delta \theta_{0} \sin (2 \pi x / L)\left[Q(t)+k_{1} e^{-t / \tau_{q}}\right],
$$

where $k_{1}=k \tau_{\theta} / \tau_{q}, k_{2}=k-k_{1}$, and

$$
\begin{aligned}
& Q\left(t ; \lambda_{i}\right)= \\
& \begin{cases}\frac{e^{\lambda_{1} t}}{\lambda_{2}} \frac{k}{\tau_{q}} \sin \lambda_{2} t, & \text { if } \lambda_{2}^{2}>0, \\
\frac{e^{\lambda_{1} t}}{2 \lambda_{3}}\left[\left(k_{1}+\frac{k_{2}}{\tau_{q}\left(\lambda_{1}+\lambda_{3}\right)+1}\right)\left(\lambda_{3}-\lambda_{1}\right) e^{\lambda_{3} t}+\right. & \\
\left.\left(k_{1}+\frac{k_{2}}{\tau_{q}\left(\lambda_{1}-\lambda_{3}\right)+1}\right)\left(\lambda_{3}+\lambda_{1}\right) e^{-\lambda_{3} t}\right], & \text { if } \lambda_{2}^{2}<0, \\
-e^{\lambda_{1} t}\left(k_{1}+\frac{k_{2}}{\tau_{q} \lambda_{1}+1}\right) \lambda_{1} t, & \text { if } \lambda_{2}^{2}=0 .\end{cases}
\end{aligned}
$$

\footnotetext{
${ }^{3}$ It can be shown that despite the change in the integration bounds, the Jeffreys-type model in Eq. (9) is still obtained by differentiating Eq. (31) with respect to time $t$.
} 
Next, we introduce the cosine average of temperature over the length of the nanobeam:

$$
\bar{\theta}(t):=\frac{2}{L} \int_{0}^{L} \theta(x, t) \cos (2 \pi x / L) d x .
$$

Substituting $\theta$ from Eq. (30) into Eq. (35), we obtain

$$
\bar{\theta}(t) / \Delta \theta_{0}=T(t), \quad t \geq 0 .
$$

Similarly, we introduce the sine average of the $x$-component of heat flux vector:

$$
\bar{q}(t):=\int_{0}^{L} q(x, t) \sin (2 \pi x / L) d x .
$$

Substituting $q$ from Eq. (32) into Eq. (37), we obtain

$$
\bar{q}(t) / \pi \Delta \theta_{0}=Q(t)+k_{1} e^{-t / \tau_{q}}, \quad t \geq 0 .
$$

The CV model has similar solutions for $\bar{\theta}(t)$ and $\bar{q}(t)$ as in Eqs. (36) and (38) with different coefficients $\lambda_{i} ; i=1,2,3$ obtained by setting $\tau_{\theta}=0$ in Eq. (29). For the Fourier model, we have

$$
\begin{aligned}
\bar{\theta}(t) / \Delta \theta_{0}=e^{-\omega^{2} \alpha t}, & t \geq 0, \\
\bar{q}(t) / \pi \Delta \theta_{0}=k e^{-\omega^{2} \alpha t}, & t \geq 0,
\end{aligned}
$$

where $\omega=2 \pi / L$.

The binning procedure in Section 3.1 is used to obtain the temperature and heat flux of each bin at discrete time intervals. The resulting spatiotemporal temperature and heat flux profiles are used to calculate $\bar{\theta}(t)$ and $\bar{q}(t)$ using Eqs. (35) and (37). Then, the nonlinear function $T\left(t ; \lambda_{i}\right)$ in Eq. (28) is fit to the calculated dependent variable $y=\bar{\theta}(t) / \Delta \theta_{0}$ appearing in Eq. (36) to calculate the model parameter vector $\boldsymbol{\lambda}=\left(\lambda_{1}, \lambda_{2}, \lambda_{3}\right)$. Thus we have $y_{m}$ at each time $t_{m}=m \Delta_{t}\left(m=1,2, \ldots, N_{\text {profiles }}\right)$, where $N_{\text {profiles }}$ is the number of stored temperature profiles. Assuming the parameters $\boldsymbol{\lambda}$ are constant over the range of temperatures being explored, the following relationship exists between $y_{m}$ and $t_{m}$,

$$
y_{m}=T\left(t_{m} ; \boldsymbol{\lambda}\right)+R_{m}
$$


where $R_{m}$ are residual terms representing the noise in the data. In order to obtain the parameters $\boldsymbol{\lambda}$, a nonlinear regression method is used to minimize the sum of the square of the residuals:

$$
\min _{\boldsymbol{\lambda}} \sum_{m} R_{m}(\boldsymbol{\lambda})^{2}=\min _{\substack{\boldsymbol{\lambda} \\ \forall i=1,2,3}} \sum_{m}\left(y_{m}-T_{i}\left(t_{m} ; \boldsymbol{\lambda}\right)\right)^{2}
$$

where $T_{i}\left(t_{m}\right) ; i=1,2,3$, corresponds to $T(t)$ for the three different cases of Eq. (28) depending upon whether $\lambda_{2}^{2}$ is positive, negative or zero. The value of $\lambda_{2}$ is unknown a priori, therefore we minimize the sum of residuals for all three cases and select the $\lambda_{2}$ value for which the sum of residuals is minimal.

Once the parameters $\lambda_{i}$ are known, Eq. (29) provides a system of two equations for the three unknowns $\alpha=k / \gamma, \tau_{\theta}$ and $\tau_{q}$. If we are fitting the CV model, then $\tau_{\theta}=0$, and we can solve for the remaining coefficients:

$$
k=\gamma\left(\lambda_{1}^{2}+\lambda_{2}^{2}\right) \frac{\tau_{q}}{\omega^{2}}, \quad \tau_{q}=-\frac{1}{2 \lambda_{1}} .
$$

For the Jeffreys-type model, we can solve for $k$ and $\tau_{\theta}$ in terms of $\tau_{q}$ :

$$
k=\gamma\left(\lambda_{1}^{2}+\lambda_{2}^{2}\right) \frac{\tau_{q}}{\omega^{2}}, \quad \tau_{\theta}=-\frac{1+2 \tau_{q} \lambda_{1}}{\left(\lambda_{1}^{2}+\lambda_{2}^{2}\right) \tau_{q}} .
$$

Substituting these relations and the values of $\lambda_{i}$ into Eq. (34), we rewrite $Q=\widehat{Q}\left(t ; \tau_{q}\right)$ in terms of $\tau_{q}$. Thus Eq. (38) becomes

$$
\bar{q}\left(t ; \tau_{q}\right) / \pi \Delta \theta_{0}=\tilde{Q}\left(t ; \tau_{q}\right), \quad t \geq 0,
$$

where $\tilde{Q}\left(t ; \tau_{q}\right)=\widehat{Q}\left(t ; \tau_{q}\right)-\gamma \frac{1+2 \tau_{q} \lambda_{1}}{\omega^{2} \tau_{q}} e^{-t / \tau_{q}}$. In order to determine the parameter $\tau_{q}$, we fit the nonlinear function $\tilde{Q}\left(t ; \tau_{q}\right)$ to the dependent variable $z=\bar{q} / \pi \Delta \theta_{0}$ appearing in Eq. (45). This leads to the following relation,

$$
z_{m}=\tilde{Q}\left(t_{m} ; \tau_{q}\right)+\tilde{R}_{m}
$$

where $z_{m}$ is the value of the dependent variable at time $t_{m}=m \Delta_{t}(m=$ $\left.1,2, \ldots, N_{\text {profiles }}\right)$, and $\tilde{R}_{m}$ is the corresponding residual term. Since the $\lambda_{i}$ values and the form of $\tilde{Q}$ are known, $\tau_{q}$ can be solved using a nonlinear regression method:

$$
\min _{\tau_{q}} \sum_{m} \tilde{R}_{m}\left(\tau_{q}\right)^{2}=\min _{\tau_{q}} \sum_{m}\left(z_{m}-\tilde{Q}\left(t_{m} ; \tau_{q}\right)\right)^{2},
$$

subject to the constraint $\left(\tau_{q}, k, \tau_{\theta}\right) \geq(0,0,0)$. Finally, Eq. (44) is used to calculate $k$ and $\tau_{\theta}$. 


\section{Numerical results}

In order to test the methods described in Section 3, MD and NEMD simulations were carried out on argon nanobeams. The atomic interactions were modeled using a LJ pair potential [71, 72] with LJ parameters [73] $\sigma=3.4 \AA$ and $\epsilon=0.01025423 \mathrm{eV}$, and a simple truncation cutoff, i.e., $\phi(r)=0$ for $r \geq r_{\text {cut }}$, archived in OpenKIM [74-76]. The cutoff radius was taken to be $r_{\text {cut }}=4.5 \sigma=15.3 \AA$. Temperature BCs were applied using either a NoséHoover chain thermostat [77 82] or a Langevin thermostat [65, 83] using a definition for the temperature which is invariant with respect to rigid-body motions due to Jellinek and Li [63]. The parallel MD code LAMMPS [84, 85] was used to integrate the equations of motion with the following modifications: (a) temperature calculations employed the Jellinek and Li [63] approach mentioned above; (b) the binning procedure of Section 3.1 was used instead of a similar feature in LAMMPS called "layers" "4; and (c) heat-flux was computed for each bin after removing rigid-body translation 5

\subsection{Initialization}

All simulations begin with an initialization stage during which the system is equilibrated to the initial target temperature $\theta_{0}$ and allowed to expand to its equilibrium volume. The argon nanobeam has an fcc structure and is oriented so that the $x, y$ and $z$ axes coincide with the crystallographic [100], [010] and [001] directions. A nanobeam of size $n_{x} a_{0} \times n_{y} a_{0} \times n_{z} a_{0}$ (with $n_{y}=n_{z}$ ) is constructed, where $a_{0}=5.26 \AA$ is the zero-temperature equilibrium constant for argon. Periodic BCs are applied in all directions. The initial momenta of all the atoms are selected from a Maxwell-Boltzmann distribution for twice the target temperature $\left(2 \theta_{0}\right)$. This is done because approximately half of the initial kinetic energy is transformed to potential energy due to the equipartition of energy, and so the final temperature after equilibration will be about $\theta_{0}$. The velocities of the atoms are adjusted to ensure that the system has zero linear and angular momentum. After assigning the initial velocities, the nanobeam is evolved subject to NPT conditions

\footnotetext{
${ }^{4}$ In the LAMMPS implementation the number of layers (bins) changes during the simulation, which is undesirable for the TPI approach.

${ }^{5}$ It was observed that the removal of rigid-body rotation did not significantly affect the temperature and heat-flux values; therefore in order to save computational time only rigid-body translation was removed in most cases.
} 
Table 1: Lattice constants $a(\AA)$ at different temperatures $\theta_{0}(\mathrm{~K})$.

\begin{tabular}{cccccccccc}
\hline \hline$\theta_{0}$ & $a$ & $\theta_{0}$ & $a$ & $\theta_{0}$ & $a$ & $\theta_{0}$ & $a$ & $\theta_{0}$ & $a$ \\
5 & 5.262 & 10 & 5.267 & 15 & 5.278 & 20 & 5.284 & 25 & 5.291 \\
30 & 5.312 & 40 & 5.338 & 50 & 5.364 & 60 & 5.394 & 70 & 5.430 \\
\hline \hline
\end{tabular}

with the pressure set to zero and the temperature set to $\theta_{0}$. A coupled NoséHoover chain thermostat (chain length $=3$ ) and barostat as described in Shinoda et al. 86] were used. This method combines the hydrostatic equations of Tobias et al. [87] with the strain energy proposed by Parrinello and Rahman [88]. The method is implemented within the LAMMPS code. The damping times defined by LAMMPS for the thermostat and barostat were taken to be $100 \mathrm{fs}$ and $400 \mathrm{fs}$, respectively. The system is evolved for a total of $3 \times 10^{6} \mathrm{MD}$ steps with a time step size $\Delta t_{\mathrm{MD}}=2 \mathrm{fs}$. The lattice constant at the target temperature $\theta_{0}$ is determined by computing the average volume $\bar{V}$ over the final $10^{4}$ MD steps, then $a=\left[\bar{V} /\left(n_{x} n_{y} n_{z}\right)\right]^{1 / 3}$. The averaging is necessary because under NPT conditions the volume fluctuates about the mean value. Lattice constants at different temperatures obtained using this procedure are presented in Table 1 .

\subsection{Green-Kubo method}

Following the equilibration procedure of the previous section, an $N V T$ simulation is performed for $2 \times 10^{6} \mathrm{MD}$ steps followed by an $N V E$ simulation for $6 \times 10^{6} \mathrm{MD}$ steps, with a time step size of $\Delta t_{\mathrm{MD}}=1 \mathrm{fs}$. The lack of a thermostat means that the system temperature will oscillate about the target temperature, however, this approach ensures that perturbations caused by thermostats do not affect the computation of the heat current autocorrelation function. The NVE data is divided into six groups each corresponding to 1 ns of simulation time. The thermal conductivity tensor for each group, as a function of the integration time $\tau_{I}$ is computed using Eq. (15) with the first $5 \times 10^{5}$ steps excluded. The statistical error is estimated using the approach described in Ref. [38]. The thermal conductivity $k$ of the system is taken to be the average of the diagonal components of the ensemble average of the tensor. This value oscillates about a plateau within a range of $\tau_{I}$ (see Fig. (4). Finally, a mean of all thermal conductivities computed within this range along with the maximum standard error is reported. (See the tabulated results presented below.) 


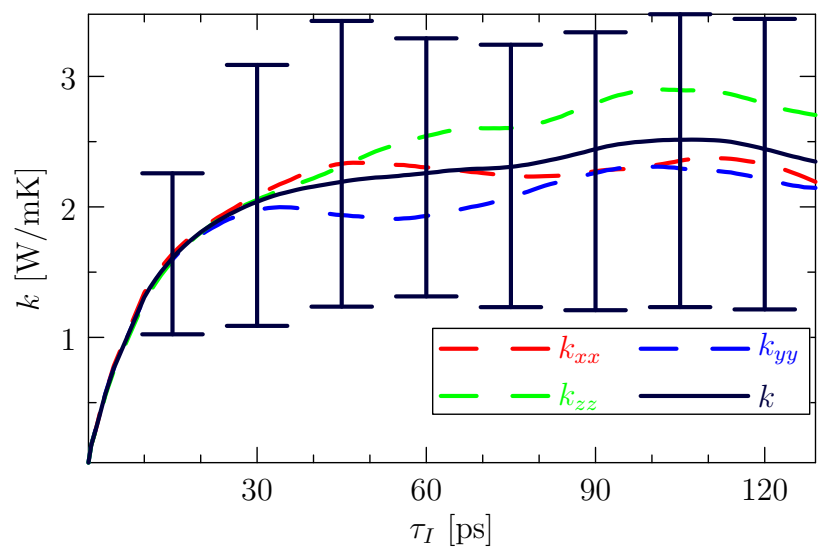

Figure 4: Thermal conductivity tensor diagonal components and their average, $k$, as a function of the integration time $\tau_{I}$ for a beam of size $33 \times 4 \times 4$ unit cells at $\theta_{0}=15 \mathrm{~K}$ subject to periodic BCs. Within the range $50 \mathrm{ps} \leq \tau_{I} \leq 100 \mathrm{ps}, k$ oscillates around a plateau.

\subsection{Direct method}

In the direct method, a temperature gradient is imposed along the length of the beam by thermostatting certain regions of the beam. The beam is partitioned into bins by the method described in the Section 3.1. As a typical example, consider a beam of size $123 \times 4 \times 4$ unit cells with $\theta_{\mathrm{L}}=\theta_{\mathrm{R}}=$ $12.5 \mathrm{~K}$ and $\theta_{\mathrm{M}}=17.5 \mathrm{~K}$. The beam is divided into $N_{\text {bins }}=41$ bins with each bin consisting of 192 atoms. The bins at the two ends and in the middle are thermostatted by Langevin dynamics [83]. The initial lattice parameter is taken to be the average of values computed for the end bins and middle bin temperatures (see Table 11). Figure 5 presents the temperature and heat current profiles at steady state for different damping times used in the Langevin dynamics. The damping time $t_{\text {damp }}$ is akin to a relaxation time which is normally inversely proportional to the viscosity of the medium. A damping time with a value of 1.0 means that during the thermostatting process, temperature is relaxed over a time span of roughly 1 ps.

From Fig. 5(a) it is clear that the temperature profiles are piecewise linear with discontinuous jumps between thermostatted and adjacent unthermostatted bins. These jumps are due to the artificial Kapitza effect discussed in the introduction. Standard errors in thermal conductivity are calculated using the method of Zhou et al. [69]. Details are given in the supplementary online material. 


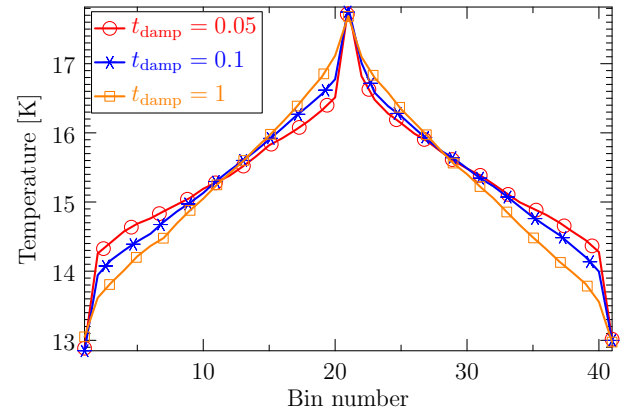

(a)

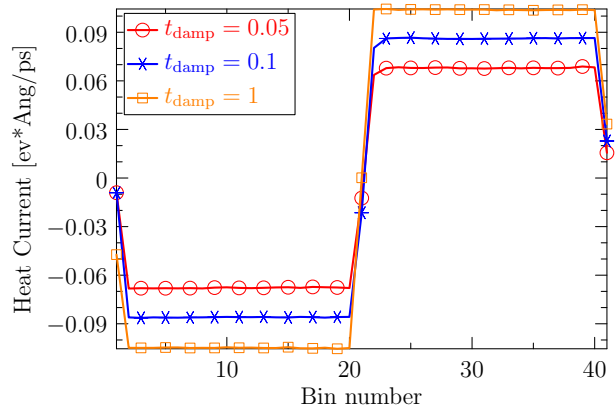

(b)

Figure 5: Profiles of steady-state (a) temperature and (b) heat current along the length of the beam for different damping times under periodic BCs for an argon beam of size $123 \times 4 \times 4$ unit cells.

\subsection{Thermal parameter identification method}

We illustrate the TPI method for a beam of size $153 \times 4 \times 4$ unit cells. The initialization stage is first performed as outlined in Section 4.1 to bring the beam to steady state at the target temperature of $\theta_{0} \approx 15 \mathrm{~K}$. Then binning is performed with a total number of $N_{\text {bins }}=51$ bins such that each bin contains 192 atoms (see Section 3.1). Next the sinusoidal temperature distribution in Eq. (21) is applied by setting the temperature of the $n$-th bin to

$$
\theta_{n}=\theta_{0}+\Delta \theta_{0} \cos \left(2 \pi n / N_{\text {bins }}\right), \quad 1 \leq n \leq N_{\text {bins }},
$$

where $\Delta \theta_{0}=0.1 \theta_{0}$, using a Langevin thermostat with a damping time of 1 ps. Thus $N_{\text {bins }}$ thermostats with the same damping time (but different temperatures) are employed. The system is run for $2 \times 10^{6} \mathrm{MD}$ steps with a time step of $\Delta t_{\mathrm{MD}}=2 \mathrm{fs}$. Having attained the sinusoidal temperature distribution, the system is run for another $5 \times 10^{5}$ MD steps, during which the positions and momenta of all atoms are stored at intervals of $10^{4} \mathrm{MD}$ steps. Thus 50 independent realizations satisfying the initial conditions described by Eq. (48) are obtained for further relaxation of the system. For each realization, the thermostats are switched off and the system is left to evolve under $N V E$ conditions for another $56 \times 10^{4} \mathrm{MD}$ steps with a time step of $\Delta t_{\mathrm{MD}}=1 \mathrm{fs}$. The temperature and $x$-component of the heat flux current for each bin, $\theta_{n}$ and $J_{x_{n}}$, are recorded, using Eqs. (12) and (16), and averaged over $\Delta_{t}=1.4$ ps using Eq. (13). Thus $N_{\text {profiles }}=400$ temperature and heat current profiles are collected. We vary $\Delta_{t}$ for different lengths so that before 


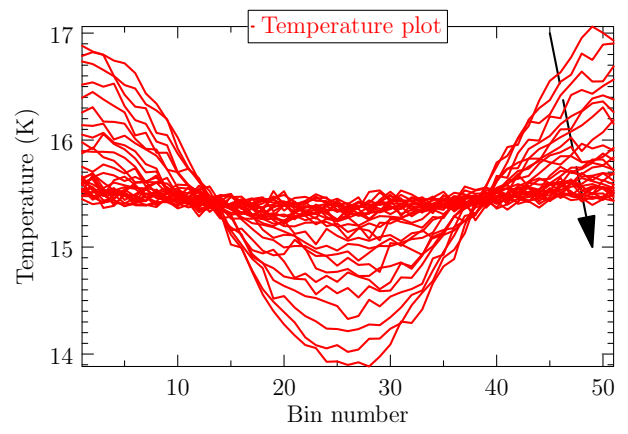

(a)

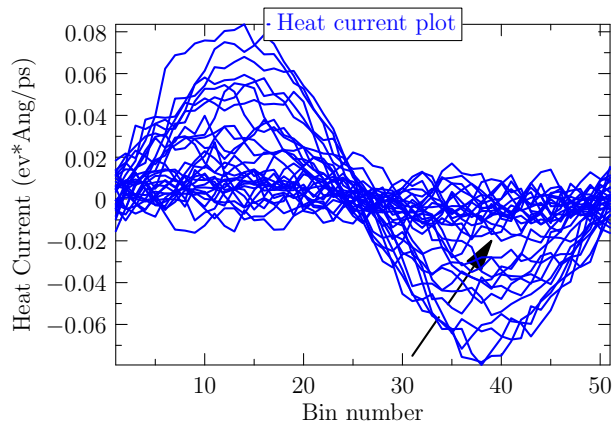

(b)

Figure 6: Temperature and heat current profiles for an argon beam of size $153 \times 4 \times 4$ unit cells with the base temperature $\theta_{0} \approx 15 \mathrm{~K}$ and the amplitude of sinusoidal perturbation $\Delta \theta_{0} \approx 1.5 \mathrm{~K}$. Each profile is an average of $14 \mathrm{ps}$ of data. The black arrows show the direction of time.

reaching the steady-state we collect enough profiles to analyze the nonsteady relaxation behavior of the system. Thermal fluctuations are further reduced by averaging over multiple realizations. Averaging over all 50 realizations, the temperature and heat current profiles are shown in Fig. 6. Next, we calculate $\bar{\theta}(t)$ and $\bar{q}(t)$ using Eqs. (35) and (37) for these profiles 6 and perform the nonlinear least square fitting of functions $\bar{\theta} / \Delta \theta_{0}$ and $\bar{q}(t) / \pi \Delta \theta_{0}$ as defined in Eqs. (42) and (47), respectively. The plots of these functions are shown in Fig. (7. The expression of $\bar{\theta}(t) / \Delta \theta_{0}$ in Eq. (36) is same for both the CV and the Jeffreys-type model, and these models successfully capture the NEMD data. However, we note that the NEMD data includes a short wave length oscillation with a periodicity of about $50 \mathrm{ps}$. In order to investigate the origin of this oscillation, we calculate the cosine average of the stress component $\sigma_{x x}$ over length of the beam:

$$
\bar{\sigma}_{x x}(t)=\frac{2}{L} \int_{0}^{L} \sigma_{x x}(x, t) \cos (2 \pi x / L) d x .
$$

The plot of $\bar{\sigma}_{x x}(t)$ with respect to time $t$ and its periodogram power spectral density (PSD) are shown in Fig. 8. The PSD has a dominant spike at a frequency of $0.018 \mathrm{ps}^{-1}$, which suggests that the system has a stress wave of

\footnotetext{
${ }^{6}$ We have explored the effect of the number of profiles used in computing Eqs. (35) and (37). We found that increasing the number of bins had only a small effect (less than the standard errors) on the final values of the parameters.
} 


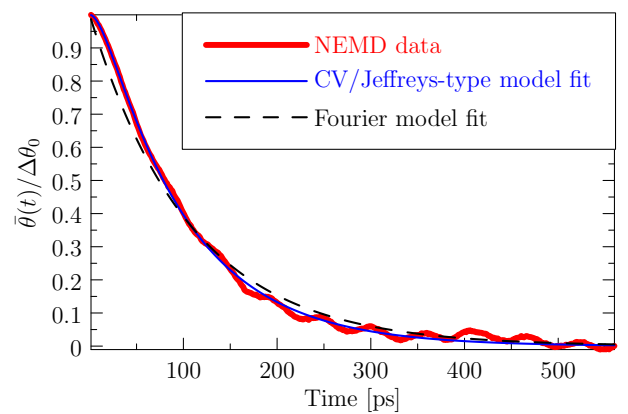

(a)

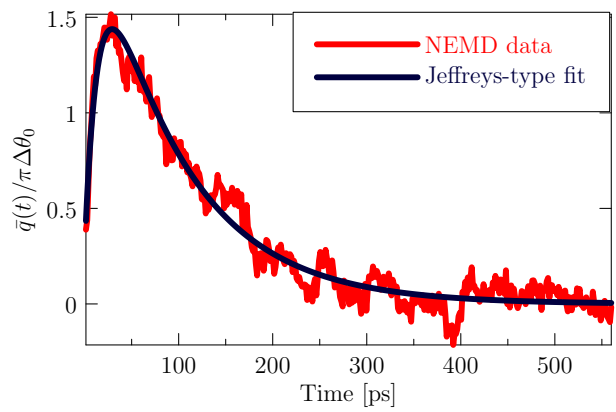

(b)

Figure 7: (a) Plot of $\bar{\theta}(t) / \Delta \theta_{0}$ with respect to time $t$ for the beam under study. The red, blue and black lines correspond to NEMD data, CV/Jeffreys-type model and Fourier model, respectively. (b) Plot of $\bar{q}(t) / \pi \Delta \theta_{0}$ with respect to time $t$. The red and dark blue lines correspond to NEMD data and Jeffreys-type model, respectively.

period 55.14 ps. This is very close to the oscillation period present in the $\bar{\theta}(t) / \Delta \theta_{0}$ plot. Moreover, the longitudinal speed of sound in solid argon at $15 \mathrm{~K}$ is around [89] $1600 \mathrm{~m} / \mathrm{s}$, thus a stress wave at this speed will travel the length of the nanobeam in about $50.5 \mathrm{ps}$. This time is also very close to the oscillation period. Hence we conclude that the system develops a very low stress wave, of the order of a few bars in magnitude, that affects $\bar{\theta}(t) / \Delta \theta_{0}$ through thermomechanical coupling and leads to the observed oscillations.

We also tried fitting the NEMD $\bar{\theta}(t) / \Delta \theta_{0}$ data with the Fourier model given in Eq. (39)). The results in Fig. 7(a) may suggest that the Fourier model adequately captures the NEMD data. However, it is clear in Fig. 7(b) that the exponentially increasing part of the $\bar{q}(t) / \pi \Delta \theta_{0}$ curve (near $t=0$ ) cannot be captured by the exponentially decaying expression for the Fourier model in Eq. (40). In contrast, the expression for the Jeffreys-type model given in Eq. (38) provides a good fit to the NEMD data.

The volumetric heat capacity $\gamma$ appearing in Eqs. (43) and (44) is computed in a separate calculation. The entire beam is brought to steady state at $\theta=15 \mathrm{~K}$ with a Langevin thermostat for $2 \times 10^{6} \mathrm{MD}$ steps with a time step size $\Delta t_{\mathrm{MD}}=2 \mathrm{fs}$. The beam continues to be thermostatted for another $3 \times 10^{5}$ MD steps and the energy of the beam is recorded at each MD step. Then $\gamma$ is related to the average energy fluctuations through the following relation [90]:

$$
k_{\mathrm{B}} \theta^{2} \gamma V=\left\langle E^{2}\right\rangle-\langle E\rangle^{2},
$$




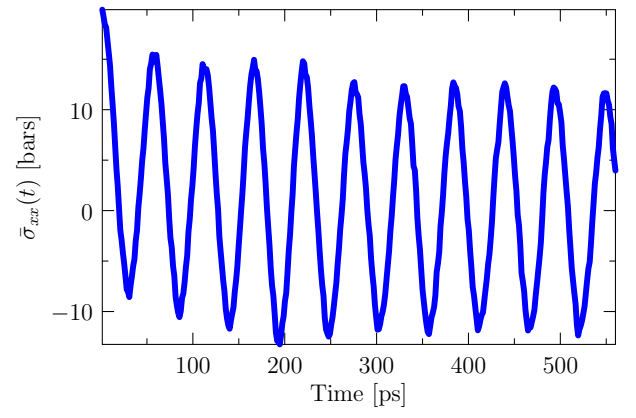

(a)

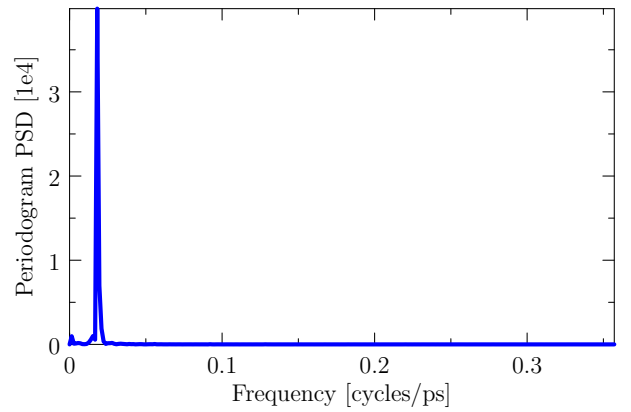

(b)

Figure 8: (a) $\bar{\sigma}_{x x}(t)$ vs time $t$, (b) Periodogram power spectral density estimate of the signal $\sigma_{x x}^{-}(t)$ about its mean value.

where $k_{\mathrm{B}}$ is Boltzmann's constant. Using Eq. (50), we obtain $\gamma=7.14 \pm 0.04 \times$ $10^{-6} \mathrm{eV} /\left(\AA^{3} \mathrm{~K}\right)$. We find that $\gamma$ does not change significantly with system size. Substituting $\gamma$ into Eqs. (43) and (44), the following results are obtained for the $\mathrm{CV}$ parameters of argon, $k^{\mathrm{CV}}=1.72 \mathrm{~W} / \mathrm{mK}$ and $\tau_{q}^{\mathrm{CV}}=12.11 \mathrm{ps}$, and the Jeffreys parameters for argon, $k=1.75 \mathrm{~W} / \mathrm{mK}, \tau_{q}=12.33 \mathrm{ps}$, and $\tau_{\theta}=1.94 \mathrm{ps}$. In order to calculate standard errors in these parameters, we partition all 50 realizations into 4 sets and perform the nonlinear least square fitting over each set separately.

Table 2 presents the values of the thermal conductivity for different temperatures and beam lengths under periodic BCs obtained by the Green-Kubo and NEMD direct methods. Tables 3 ,5 present the thermal parameters for the Fourier, CV and Jeffreys-type models obtained using the TPI method. We see that the thermal conductivities obtained for all three models are nearly the same, but the relaxation times vary.

A striking result is that at a given temperature, the thermal parameters obtained using the direct and TPI methods are length (size) dependent. After a critical length [70], the dependence of thermal conductivity $k$ on length $L$ takes the following form [38, 50, 70]:

$$
\frac{1}{k}=\frac{1}{k_{\infty}}+\frac{c}{L}
$$

where $c$ is a constant dependent on the method of calculation, and $k_{\infty}$ is the bulk value. Fig. $9(\mathrm{a})$ presents a plot of $1 / k$ versus $1 / L$ at $\theta_{0}=15 \mathrm{~K}$ for three different methods: (i) Green-Kubo method, (ii) direct method, 
Table 2: Thermal conductivity $k$ in $\mathrm{W} / \mathrm{mK}$ obtained using the Green-Kubo and direct method for different beam lengths $L$ in unit cells and different temperature. Values in parentheses are the standard errors of the thermal conductivities.

\begin{tabular}{cccccc}
\hline \hline \multicolumn{6}{c}{ Green-Kubo method under periodic BCs } \\
\multicolumn{5}{c}{ Temp (K) } \\
$L$ & 15 & 25 & 35 & 45 & 55 \\
\hline 33 & $2.34(1.10)$ & $1.06(0.52)$ & $0.72(0.20)$ & $0.51(0.31)$ & $0.35(0.09)$ \\
63 & $2.67(2.48)$ & $1.14(0.44)$ & $0.74(0.20)$ & $0.47(0.20)$ & $0.36(0.17)$ \\
93 & $2.58(1.24)$ & $1.10(0.61)$ & $0.73(0.29)$ & $0.47(0.18)$ & $0.35(0.19)$ \\
153 & $2.89(1.85)$ & $1.07(0.21)$ & $0.81(0.26)$ & $0.46(0.13)$ & $0.36(0.10)$ \\
303 & $2.28(1.69)$ & $1.08(0.79)$ & $0.70(0.23)$ & $0.51(0.40)$ & $0.34(0.11)$ \\
603 & $2.52(1.91)$ & $1.09(0.65)$ & $0.74(0.25)$ & $0.51(0.31)$ & $0.35 \mathrm{i}(0.15)$ \\
\hline \multicolumn{5}{c}{ Direct method under periodic BCs } \\
\multicolumn{5}{c}{ Temp (K) } \\
$L$ & 15 & 25 & 35 & \\
\hline 33 & $1.82(0.06)$ & $0.73(0.02)$ & $0.61(0.04)$ & $0.40(0.01)$ & $0.32(0.02)$ \\
63 & $2.06(0.02)$ & $1.05(0.04)$ & $0.65(0.01)$ & $0.49(0.03)$ & $0.34(0.04)$ \\
93 & $2.16(0.02)$ & $1.12(0.01)$ & $0.68(0.03)$ & $0.50(0.01)$ & $0.37(0.04)$ \\
153 & $2.22(0.03)$ & $1.11(0.03)$ & $0.68(0.03)$ & $0.50(0.02)$ & $0.41(0.03)$ \\
303 & $2.33(0.04)$ & $1.18(0.01)$ & $0.69(0.02)$ & $0.50(0.02)$ & $0.39(0.04)$ \\
603 & $2.34(0.06)$ & $1.13(0.03)$ & $0.70(0.03)$ & $0.50(0.02)$ & $0.40(0.04)$ \\
\hline \hline
\end{tabular}

Table 3: The Fourier thermal conductivity $k$ in units of $\mathrm{W} / \mathrm{mK}$ at different temperatures obtained using the TPI method by nonlinear fitting of the expression for $\bar{\theta}(t) / \Delta \theta_{0}$ in Eq. (39). Values in parentheses are the standard errors of the parameters.

\begin{tabular}{ccccc}
\hline \hline \multicolumn{5}{c}{ Fourier thermal conductivity } \\
Tength & 15 & 25 & 35 & 45 \\
\hline 33 & $0.75(0.06)$ & $0.46(0.02)$ & $0.36(0.05)$ & $0.29(0.02)$ \\
63 & $0.99(0.07)$ & $0.65(0.03)$ & $0.48(0.03)$ & $0.36(0.07)$ \\
93 & $1.19(0.03)$ & $0.83(0.07)$ & $0.50(0.01)$ & $0.38(0.02)$ \\
153 & $1.71(0.08)$ & $0.87(0.04)$ & $0.56(0.02)$ & $0.44(0.04)$ \\
303 & $2.09(0.09)$ & $1.06(0.05)$ & $0.60(0.01)$ & $0.47(0.03)$ \\
603 & $2.32(0.03)$ & $1.24(0.03)$ & $0.75(0.03)$ & $0.51(0.02)$ \\
\hline \hline
\end{tabular}


Table 4: The CV model parameters at different temperatures obtained using the TPI method by nonlinear fitting of the expression for $\bar{\theta}(t) / \Delta \theta_{0}$ in Eq. (36). For each length, the two rows are the values of thermal conductivity $k^{\mathrm{CV}}$ in $\mathrm{W} / \mathrm{mK}$ and the relaxation time $\tau_{q}^{\mathrm{CV}}$ in ps. Values in parentheses are the standard errors of the parameters.

\begin{tabular}{ccccc}
\hline \hline \multicolumn{5}{c}{ CV parameters } \\
Temp $(\mathrm{K})$ \\
Length & 15 & 25 & 35 & 45 \\
\hline \multirow{3}{*}{33} & $0.75(0.15)$ & $0.47(0.02)$ & $0.37(0.04)$ & $0.29(0.02)$ \\
& $1.83(1.18)$ & $0.63(0.84)$ & $2.00(2.59)$ & $2.43(2.24)$ \\
\hline \multirow{6}{*}{63} & $1.00(0.09)$ & $0.65(0.03)$ & $0.48(0.03)$ & $0.36(0.07)$ \\
& $2.90(2.38)$ & $1.21(1.42)$ & $4.07(4.43)$ & $3.56(3.71)$ \\
\hline \multirow{5}{*}{93} & $1.20(0.03)$ & $0.83(0.07)$ & $0.50(0.01)$ & $0.38(0.02)$ \\
& $4.62(3.81)$ & $5.58(2.06)$ & $6.35(2.35)$ & $3.29(2.87)$ \\
\hline \multirow{6}{*}{153} & $1.73(0.09)$ & $0.89(0.01)$ & $0.56(0.02)$ & $0.44(0.04)$ \\
303 & $12.79(6.81)$ & $9.02(8.31)$ & $7.41(6.08)$ & $17.69(8.49)$ \\
\hline 603 & $19.09(0.10)$ & $1.06(0.05)$ & $0.60(0.01)$ & $0.47(0.04)$ \\
\hline \hline
\end{tabular}


Table 5: Parameters at different temperatures obtained with the TPI method by nonlinear fitting of the expression for $\bar{\theta}(t) / \Delta \theta_{0}$ in Eq. (36) and $\bar{q}\left(t ; \tau_{q}\right) / \pi \Delta \theta_{0}$ in Eq. (45). The first column is the beam length in unit cells. For each length, the three rows are the values of thermal conductivity $k$ in $\mathrm{W} / \mathrm{mK}$ and the two time lags $\tau_{\theta}$ and $\tau_{q}$ in ps. Values in parentheses are the standard errors of the parameters.

\begin{tabular}{ccccc}
\hline \hline \multicolumn{5}{c}{ Jeffreys-type parameters } \\
Length & 15 & 25 & 35 & 45 \\
\hline \multirow{3}{*}{33} & $0.74(0.14)$ & $0.49(0.09)$ & $0.36(0.06)$ & $0.33(0.03)$ \\
& $0.53(0.45)$ & $0.15(0.10)$ & $1.55(1.15)$ & $1.93(2.13)$ \\
& $2.59(0.40)$ & $1.90(1.23)$ & $5.27(0.05)$ & $4.69(2.15)$ \\
\hline \multirow{4}{*}{63} & $1.04(0.14)$ & $0.70(0.08)$ & $0.48(0.03)$ & $0.35(0.03)$ \\
& $0.59(0.13)$ & $1.49(1.23)$ & $0.66(0.58)$ & $4.84(3.45)$ \\
& $4.90(0.30)$ & $3.81(2.41)$ & $2.76(1.35)$ & $4.98(4.39)$ \\
\hline \multirow{4}{*}{93} & $1.22(0.05)$ & $0.86(0.08)$ & $0.52(0.02)$ & $0.40(0.03)$ \\
& $1.99(2.43)$ & $2.09(1.90)$ & $4.06(3.58)$ & $2.43(2.16)$ \\
& $7.40(4.06)$ & $5.79(2.21)$ & $6.33(4.36)$ & $5.71(1.90)$ \\
\hline \multirow{3}{*}{153} & $1.77(0.12)$ & $0.92(0.01)$ & $0.59(0.03)$ & $0.46(0.06)$ \\
& $1.85(2.46)$ & $6.22(3.81)$ & $9.40(4.56)$ & $19.54(11.54)$ \\
& $13.15(7.35)$ & $13.67(6.45)$ & $12.85(3.28)$ & $22.79(5.08)$ \\
\hline \multirow{3}{*}{303} & $2.14(0.08)$ & $1.08(0.07)$ & $0.63(0.01)$ & $0.48(0.06)$ \\
& $3.62(4.21)$ & $9.11(4.48)$ & $39.69(5.09)$ & $21.45(12.21)$ \\
& $23.17(1.14)$ & $16.38(4.07)$ & $40.73(11.55)$ & $25.87(6.81)$ \\
\hline \multirow{3}{*}{603} & $2.32(0.01)$ & $1.25(0.05)$ & $0.78(0.03)$ & $0.51(0.03)$ \\
& $3.62(2.82)$ & $12.88(6.79)$ & $12.42(4.23)$ & $23.55(11.89)$ \\
& $20.34(1.90)$ & $20.45(5.90)$ & $20.81(4.28)$ & $26.56(5.11)$ \\
\hline \hline
\end{tabular}




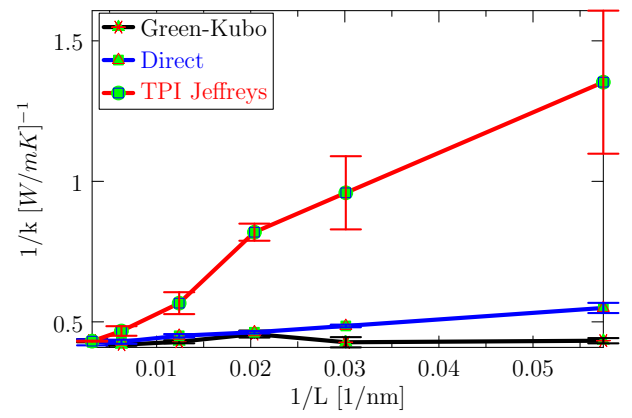

(a)

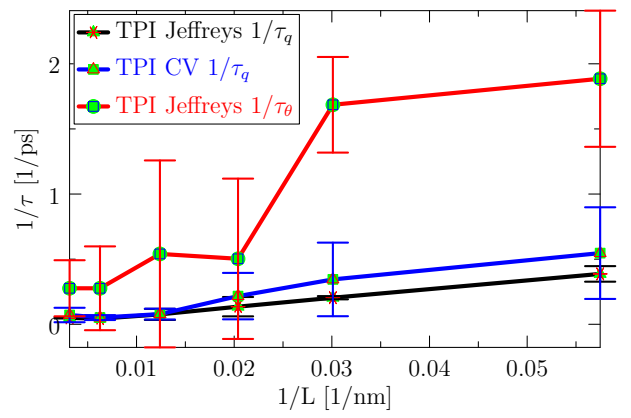

(b)

Figure 9: Plots at $\theta_{0}=15 \mathrm{~K}$ of (a) $1 / k$ versus $1 / L$ for the Green-Kubo method, direct method, and the TPI method with the Jeffreys-type model, (b) $1 / \tau$ vs $1 / L$ for the TPI method with CV and Jeffreys-type models.

(iii) TPI method for the Jeffreys-type model. Fitting the data in this plot to the relation in Eq. (51), yields $k_{\infty}$ values for the three methods which are in good agreement: $2.32 \pm 0.10,2.39 \pm 0.06,2.57 \pm 0.14 \mathrm{~W} / \mathrm{mK}$. The uncertainty reported for the bulk thermal conductivity for each method is taken as the maximum over the standard errors of the length-dependent thermal conductivities computed using that method, i.e. it is the $L^{\infty}$-norm. Not shown in Fig. 9(a), the TPI results for the Fourier and CV models give $k_{\infty}$ values that are very close to that of the TPI method for the Jeffreystype model. The temperature dependence of the bulk thermal conductivity obtained using these three methods is shown in Fig. 10. A power law fit to the data, $k_{\infty}(\theta)=\theta^{n}$, gives $n=-1.41 \pm 0.03$. The computed values are in good agreement with each other and with the MD simulation results of Kaburaki et al. [91].

Non-Fourier relaxation times also exhibit a length dependence as shown in Fig. 9(b), This effect can be understood by considering the contributions to the relaxation time due to normal and Umklapp processes of intrinsic phonon-phonon scattering phenomena [92], and applying the Matthiesen rule which assumes that all scattering mechanisms are independent. This leads to a linear relationship between $1 / \tau$ and $1 / L$ (where $\tau$ is either $\tau_{q}$ or $\tau_{\theta}$ ) similar to that obtained for thermal conductivity [70, 93]:

$$
\frac{1}{\tau}=\frac{1}{\tau_{\infty}}+\frac{c_{\tau}}{L}
$$

Here $c_{\tau}$ is a constant dependent on the model. Fitting to the data presented 


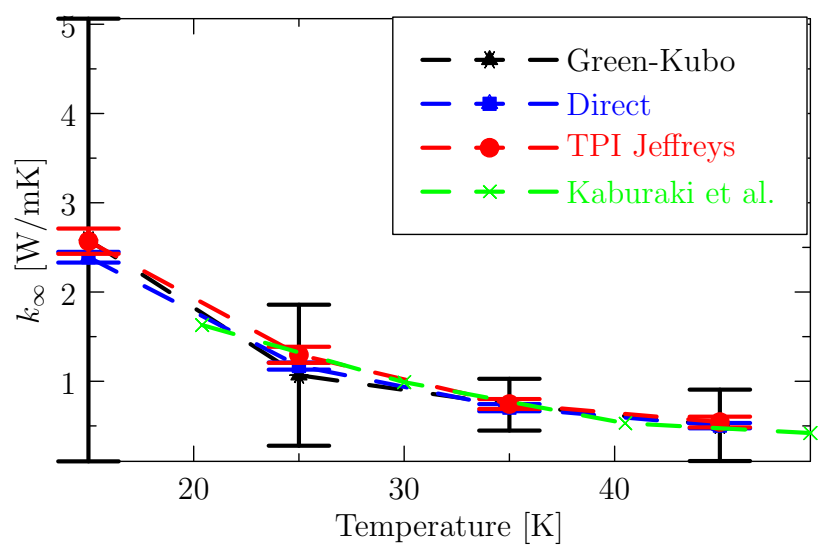

Figure 10: Bulk thermal conductivity versus temperature obtained by fitting Eq. (51) using data from the Green-Kubo method, direct method, and the TPI method with the Jeffreys-type model.

in Fig. 9(b), we find at $\theta_{0}=15 \mathrm{~K}$ that the bulk values $\tau_{\infty}$ for the Jeffreys-type relaxation times $\tau_{q}$ and $\tau_{\theta}$ are $120.36 \pm 7.35 \mathrm{ps}$ and $6.88 \pm 4.21 \mathrm{ps}$, respectively, and the bulk value for $\tau_{q}$ in the CV model is $96.65 \pm 10.92 \mathrm{ps.} \mathrm{Again,} \mathrm{the} \mathrm{bulk}$ value uncertainty is taken to be the $L^{\infty}$-norm (maximum) of the standard errors in the length-dependent data.

\section{Heat waves}

In this section, we study the propagation of heat pulses as originally done by Tsai and MacDonald [39]. The introduction of a heat pulse generates several waves that propagate at different speeds corresponding to different phonon modes, second sound waves, and diffusive components [39, 41, 94]. An argon beam of size $303 \times 4 \times 4$ is divided into 101 bins, where each bin consists of 243 atoms. Periodic BCs are employed. The beam temperature is initially set to $0 \mathrm{~K}$. The middle bin is then subjected to a heat pulse while the rest of the beam evolves under $N V E$ conditions. The heat pulse is introduced by rescaling the temperature of the middle bin at each MD time step $\left(\Delta t_{\mathrm{MD}}=2 \mathrm{fs}\right)$. For the first $100 \mathrm{MD}$ steps the temperature is linearly increased from $0 \mathrm{~K}$ to $20 \mathrm{~K}$. It is then maintained at $20 \mathrm{~K}$ for another 500 MD steps and then linearly decreased from $20 \mathrm{~K}$ to $0 \mathrm{~K}$ over $100 \mathrm{MD}$ steps. The temperature rescaling is done by adjusting the momenta of all the atoms 


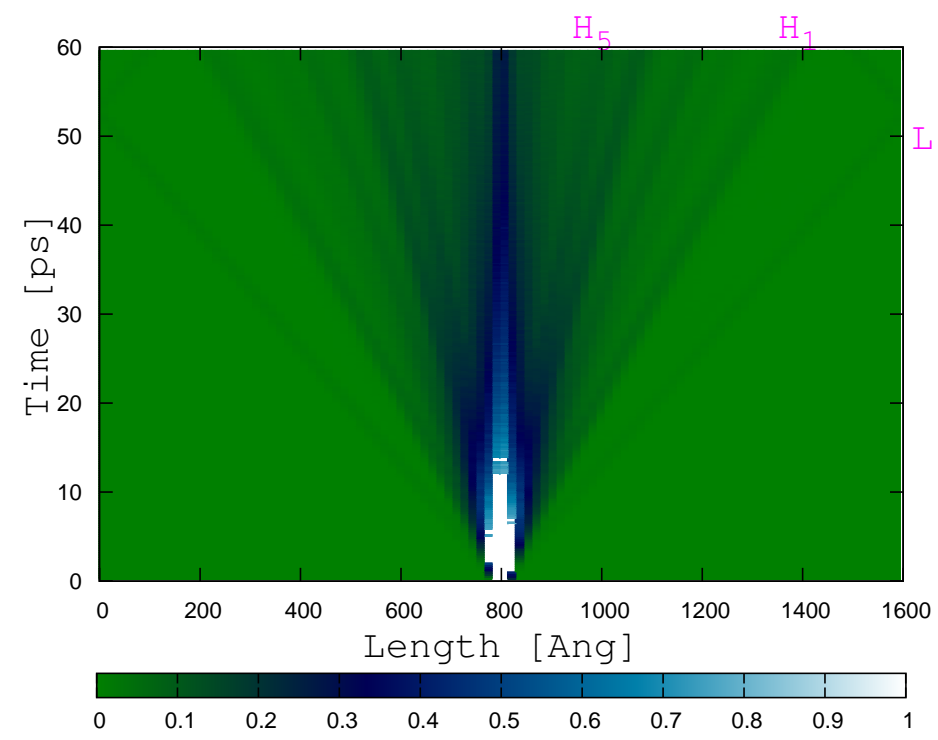

Figure 11: A plot of the temperature along the length of the beam as a function of time showing the following modes: $L$ and $H_{1}$ to $H_{5}$. The displayed temperatures are limited to a range of 0 to $1 \mathrm{~K}$ to make the presence of the waves more clear. (Any temperature larger than $1 \mathrm{~K}$ appears white.)

in the middle bin by

$$
\tilde{\boldsymbol{p}}_{i}\left(t+\Delta_{t}\right)=\tilde{\boldsymbol{p}}_{i}(t) \sqrt{\frac{\theta_{0}}{\theta(t)}},
$$

where $\theta_{0}$ is the target temperature, $\theta(t)$ is the instantaneous temperature, and $\tilde{\boldsymbol{p}}(t)$ is the adjusted momentum after removing the total linear and angular momentum of the middle bin. This is equivalent to adding or subtracting an energy $\Delta E=\Delta E_{\text {kin }}$ to the middle bin:

$$
\Delta E_{\text {kin }}=\sum_{i=1}^{N} \frac{1}{2 m_{i}}\left(\tilde{\boldsymbol{p}}_{i}^{2}(t+\Delta t)-\tilde{\boldsymbol{p}}_{i}^{2}(t)\right),
$$

where $N$ is the total number of atoms in the middle bin and $m_{i}$ is the mass of the $i$-th atom.

Following the application of the heat pulse, the system is left to evolve under $N V E$ conditions for $3 \times 10^{4} \mathrm{MD}$ steps with a step size of $\Delta t_{\mathrm{MD}}=2 \mathrm{fs}$. 
The simulation is repeated six times with different initial velocities drawn from a Maxwell-Boltzmann distribution for the initial temperature. Figure 11 shows a plot of the temperature along the length of the beam as function of time following the heat pulse. The temperature data are an average of 100 MD time steps and six ensembles corresponding to different initial velocities.

The presence of waves is clear in Fig. 11, where $L$ indicates the longitudinal stress wave, and there are five distinct heat waves ranging from $H_{1}$ and $H_{5}$. Stress and heat waves are distinguished by comparing the longitudinal $x$ and transverse $y$ and $z$ components of temperature; heat waves have nearly the same longitudinal and transverse components whereas for stress waves these components differ. Since the disturbances propagate in straight lines, we can calculate their speeds. The calculated speed of the stress-wave $L$ is $1608 \mathrm{~m} / \mathrm{s}$ and the calculated average speed of the heat waves from $H_{1}$ to $H_{5}$ is approximately $630 \mathrm{~m} / \mathrm{s}$. Feldman and Klein [95] report the longitudinal speed of sound $c_{l}$ for solid argon at $0 \mathrm{~K}$, computed using a LJ potential, as

$$
\rho c_{l}^{2} \approx 49 \mathrm{kbar}=4.9 \mathrm{GPa},
$$

where $\rho$ is the mass density of crystal. The density in our case is $\rho=$ $2307.225 \mathrm{~kg} / \mathrm{m}^{3}$. Then from Eq. (55), we have $c_{l} \approx 1457 \mathrm{~m} / \mathrm{s}$, which is in reasonable agreement (within $9.4 \%$ ) of the longitudinal stress-wave computed from Fig. 11, thus confirming the validity of the calculation. Theoretically, the average speed of heat waves is [39] $c_{\mathrm{av}} / \sqrt{3}$, where $c_{\mathrm{av}}$ is the average speed of sound appropriate to a Debye solid. Taking [39] $c_{\mathrm{av}}=0.731 c_{l}$, the theoretical average speed of the heat wave is $c_{\mathrm{av}} / \sqrt{3} \approx 678 \mathrm{~m} / \mathrm{s}$, which is comparable to the calculated average speed found above. Thus the existence of second sound in solid argon is confirmed.

It is of interest to see whether the Jeffreys-type model with parameters obtained using TPI is able to describe the heat pulse problem discussed above. This would validate the TPI approach and demonstrate that the Jeffreystype model provides an adequate description for the thermal response of solid argon and is transferable to different thermal problems. Using the TPI approach we obtain the thermal parameters for a beam (of the same size as the heat pulse beam) with periodic BCs and $\theta_{0}=2.5 \mathrm{~K}$. Taking an experimental value [96] for $\gamma$ at $2.5 \mathrm{~K}$, the estimated parameter values are $k=0.013 \pm 0.001 \mathrm{~W} / \mathrm{mK}, \tau_{\theta}=6.21 \pm 1.03 \mathrm{ps}$, and $\tau_{q}=15.57 \pm 3.47 \mathrm{ps}$. The heat wave speed predicted by the Jeffreys-type model is $c=\sqrt{k /\left(\gamma \tau_{q}\right)}=$ $596.62 \pm 70.72 \mathrm{~m} / \mathrm{s}$. This value is comparable to the speeds calculated above 


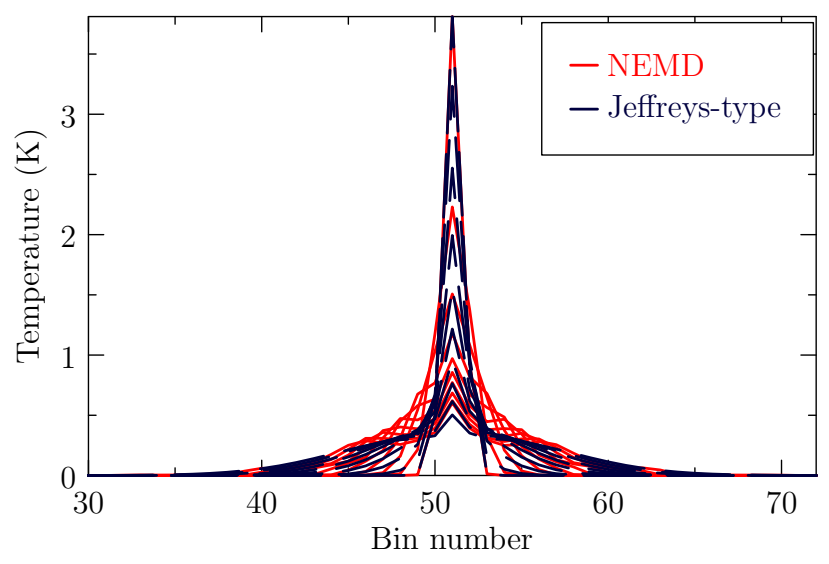

Figure 12: Comparison between NEMD data and the Jeffreys-type continuum model with thermal parameters computed using the TPI method. Each NEMD temperature profile is an average of 2 ps of NEMD data.

from the heat pulse simulation. Figure 12 compares NEMD temperature profiles with the numerical solution of the Jeffreys-type model. We see that the Jeffreys-type model with parameters obtained using the TPI method successfully describes the propagation of heat waves associated with the spreading of a thermal pulse. A similar analysis using a Fourier model (not shown) was not able to capture the sharp peaks of the temperature profiles.

\section{Conclusion}

A new simple TPI method has been proposed for extracting the thermal non-Fourier (Jeffreys-type) parameters $k, \tau_{\theta}$ and $\tau_{q}$ from a sequence of NEMD temperature and heat-flux profiles. An initial sinusoidal temperature distribution is applied using thermostats to a nanobeam. The thermostat are then removed, and the nanobeam temperature decays to a constant average value under constant energy conditions. The thermal parameters are obtained by fitting analytical solutions for scalars obtained as cosine and sine-averages of the temperature and heat flux, respectively, to the NEMD data during the decay process. (Implementations of the TPI approach in MATLAB are available in the supplementary online material.)

The TPI method was applied to argon nanobeams of various sizes at different temperatures. The values of the thermal conductivity $k$ were found to be in good agreement with those computed using the Green-Kubo and 
direct NEMD methods. In addition, the TPI method provides the relaxation times $\tau_{q}$ and $\tau_{\theta}$, which cannot be obtained using the other methods. The TPI approach also has the advantage that no thermostats are used during the calculation of the thermal parameters. It is therefore unaffected by issues, such as the artificial Kapitza effect [52], that corrupt direct method results.

It was found that a Jeffreys-type model with three parameters was better able to reproduce NEMD temperature profiles in thermal problems than a simple Fourier model. This was demonstrated by performing an NEMD simulation of the spreading of a heat pulse applied to the center of a nanobeam. The heat propagates out in waves, confirming the existence of second sound in argon. A Jeffreys-type model with parameters obtained using the TPI approach predicts heat wave speeds in agreement with the NEMD simulations and reproduces the temperature profiles to good accuracy.

\section{Appendix A. Well-Posedness of the TPI solution}

We demonstrate that the PDE in Eq. (22) is well-posed in the Hadamard sense, i.e., we show the existence, uniqueness and stability of the solution in Eq. (30).

Existence: The solution in Eq. (30) is infinitely differentiable as $\sin \lambda_{2} t$, $\cos \lambda_{2} t, e^{\lambda_{1} t}, e^{\lambda_{3} t}, t$ are infinitely differentiable functions. From Eq. (29) we have $\lambda_{1}<0$, if $\tau_{q}>0$ and $\alpha, \tau_{\theta} \geq 0$, so $e^{\lambda_{1} t} \leq 1$. Also $\lambda_{3}<\left|\lambda_{1}\right|$, so $e^{\left(\lambda_{1}+\lambda_{3}\right) t}<1$ and $e^{\left(\lambda_{1}-\lambda_{3}\right) t}<1$. For all cases in Eq. (28), it can be easily shown that $\max _{t \geq 0} T(t)=T(0)=1$. When $\lambda_{2}^{2}>0$, we have $|\dot{T}(t)|<$ $\frac{\lambda_{1}^{2}+\lambda_{2}^{2}}{\lambda_{2}}$, and $|\ddot{T}(t)|<\frac{\lambda_{1}^{2}+\lambda_{2}^{2}}{\lambda_{2}}\left(\left|\lambda_{1}\right|+\left|\lambda_{2}\right|\right)$. When $\lambda_{2}^{2}<0$, we have $|\dot{T}(t)|<$ $\frac{\lambda_{1}^{2}-\lambda_{3}^{2}}{2 \lambda_{3}}$, and $|\ddot{T}(t)|<\frac{\lambda_{1}^{2}-\lambda_{3}^{2}}{2 \lambda_{3}}\left(\left|\lambda_{1}\right|+\lambda_{3}\right)$. And for the final case when $\lambda_{2}^{2}=$ 0, we have $\max _{t \geq 0}|\dot{T}(t)|=\left|\dot{T}\left(t=-1 / \lambda_{1}\right)\right|=\left|\lambda_{1}\right| / e$ and $\max _{t \geq 0}|\ddot{T}(t)|=$ $\left|\dot{T}\left(t=-2 / \lambda_{1}\right)\right|=\lambda_{1}^{2} / e^{2}$. Therefore, $|\theta(x, t)|,\left|\frac{\partial \theta}{\partial t}(x, t)\right|,\left|\frac{\partial^{2} \theta}{\partial t^{2}}(x, t)\right|,\left|\frac{\partial^{2} \theta}{\partial x^{2}}(x, t)\right|$, $\left|\theta \frac{\partial^{3} \theta}{\partial x^{2} \partial t}(x, t)\right|$, all are bounded in their domain $(0, L) \times(0,+\infty)$. Thus the solution and derivatives appearing in the model are bounded up and the existence of the solution is confirmed. 
Uniqueness: If $\tilde{\theta}$ is another solution to Eq. (22), then $w:=\theta-\tilde{\theta}$ solves

$$
\begin{cases}w_{t t}+\frac{1}{\tau_{q}} w_{t} & =c^{2} w_{x x}+c^{2} \tau_{\theta} w_{x x t} \\ w(x, 0) & =0, \quad 0 \leq x \leq L \\ w_{t}(x, 0) & =0, \quad 0 \leq x \leq L\end{cases}
$$

where $w_{t}=\frac{\partial w}{\partial t}, w_{t t}=\frac{\partial^{2} w}{\partial t^{2}}, w_{x x}=\frac{\partial^{2} w}{\partial x^{2}}, w_{x x t}=\frac{\partial^{3} w}{\partial x^{2} \partial t}$, and $c^{2}=\alpha / \tau_{q}$. We define the energy function as

$$
E(t):=\frac{1}{2} \int_{0}^{L}\left(w_{t}^{2}+c^{2} w_{x}^{2}\right) d x .
$$

Using periodic BCs so that $w_{x}(0, t)=w_{x}(L, t)$ and $w_{x x}(0, t)=w_{x x}(L, t)$, and integration by parts, we have $\int_{0}^{L} w_{x} w_{x t} d x=-\int_{0}^{L} w_{t} w_{x x} d x$ and $\int_{0}^{L} w_{t} w_{x x t} d x=$ $-\int_{0}^{L} w_{x t}^{2} d x$. Thus

$$
\begin{aligned}
\frac{d E}{d t} & =\int_{0}^{L}\left(w_{t} w_{t t}+c^{2} w_{x} w_{x t}\right) d x \\
& =\int_{0}^{L} w_{t}\left(w_{t t}-c^{2} w_{x x}\right) d x=\int_{0}^{L} w_{t}\left(-\frac{w_{t}}{\tau_{q}}+c^{2} \tau_{\theta} w_{x x t}\right) \\
& =-\int_{0}^{L} \frac{w_{t}^{2}}{\tau_{q}} d x-c^{2} \tau_{\theta} \int_{0}^{L} w_{x t}^{2} d x<0 .
\end{aligned}
$$

Thus for $\tau_{q}>0$ and $\tau_{\theta} \geq 0$, we have $\frac{d E}{d t} \leq 0$. This means $E(t) \leq E(0)$. Since $w(x, 0)=0$ and $w$ is continuous, we have $w_{x}(x, 0)=0$. Also, $w_{t}(x, 0)=0$. Thus, $E(0)=0$. Hence $E(t)=0$ for $t \geq 0$. This means $w_{t}(x, t)=w_{x}(x, t)=$ 0 . Consequently, $w$ is constant, and $w(x, t)=w(x, 0)=0$. This implies that the solution is unique.

Stability: If $\tilde{\theta}$ is a different solution to $\theta$ due to very small changes in the initial conditions, then as above for $w:=\theta-\tilde{\theta}$, we can show that $E(t) \leq E(0)$. We define the function $F(t):=\int_{0}^{L} w^{2} d x=\|w\|^{2}$, where $\|\cdot\|$ is the $L^{2}$-norm. Taking the derivative with respect to time, we obtain $\frac{d F}{d t}=2 \int_{0}^{L} w w_{t} d x$. Since $\int_{0}^{L}\left(w-w_{t}\right)^{2} d x \geq 0$, we have

$$
\frac{d F}{d t} \leq \int_{0}^{L}\left(w^{2}+w_{t}^{2}\right) d x \leq F(t)+2 E(t) \leq F(t)+2 E(0) .
$$


Multiplying both sides of Eq. (A.3) by $e^{-t}$, we obtain

$$
\frac{d}{d t}\left(F(t) e^{-t}\right) \leq 2 E(0) e^{-t}
$$

Integrating with respect to time over $t \in\left[0, t_{0}\right]$, we obtain

$$
F(t) \leq F(0) e^{t_{0}}+2 E(0)\left(e^{t_{0}}-1\right) .
$$

This means

$$
\begin{array}{r}
\|w(x, t)\|^{2} \leq\|w(x, 0)\|^{2} e^{t_{0}}+c^{2}\left\|w_{x}(x, 0)\right\|^{2}\left(e^{t_{0}}-1\right) \\
\leq\left(\|w(x, 0)\|^{2}+c^{2}\left\|w_{x}(x, 0)\right\|^{2}\right) e^{t_{0}} .
\end{array}
$$

Thus for any small constant $\epsilon>0$, if $\|w(x, 0)\|<\epsilon$, and $\left\|w_{x}(x, 0)\right\|<\epsilon$, then $\|w(x, t)\|^{2} \leq\left(1+c^{2}\right) e^{t_{0}} \epsilon^{2}$, or $\|w(x, t)\| \leq C \epsilon$, where $C$ is a constant. This means that the difference between the two solutions $\theta$ and $\tilde{\theta}$ is bounded. Hence, the solution is stable.

\section{Acknowledgment}

The authors were fortunate to have the opportunity to discuss heat waves with Prof. Daniel Joseph who authored an important paper [2] on this subject. Prof. Joseph passed away in 2011 and the authors would like to dedicate this paper to his memory. The authors also thank Noam Bernstein for reading the manuscript and for his helpful comments. This research was partly supported through the National Science Foundation (NSF) under Grants No. PHY-0941493 and DMR-1408211 and the Air Force Office of Scientific Research (AFOSR) under Grant No. FA9550-09-1-0157.

\section{References}

[1] E. B. Tadmor, R. E. Miller, R. S. Elliott, Continuum Mechanics and Thermodynamics, Cambridge University Press, Cambridge, 2012.

[2] D. D. Joseph, L. Preziosi, Heat waves, Rev. Mod. Phys. 61 (1) (1989) 41-73.

[3] C. L. Tien, G. Chen, Challenges in microscale conductive and radiative heat transfer, J. Heat Transfer 116 (1994) 799-807. 
[4] M. Chester, Second sound in solids, Phys. Rev. 131 (5) (1963) 20132015 .

[5] C. Cattaneo, Sulla conduzione del calore, Atti. Sem. Mat. Fis. Univ. Modena 3 (1948) 83-101.

[6] C. Cattaneo, Sur une forme de l'equation de la chaleur eliminant le paradoxe d'une propagation instantanée, C. R. Acad. Sci. Paris 247 (1958) 431-433.

[7] P. Vernotte, Les paradoxes de la theorie continue de l'equatioin de la chaleur, C. R. Acad. Sci. 246 (1958) 3154-3155.

[8] P. Vernotte, La véritable équation de la chaleu, C. R. Acad. Sci. 247 (1958) 2103-2105.

[9] B. Straughan, Heat Waves, Springer, New York, 2011.

[10] W. Kaminski, Hyperbolic heat conduction equation for materials with a nonhomogeneous inner structure, J. Heat Transfer 112 (1990) 555-560.

[11] K. Mitra, S. Kumar, A. Vedavarz, M. K. Moallemi, Experimental evidence of hyperbolic heat conduction in processed meat, J. Heat Trans. 117 (1995) 568-573.

[12] H. Herwig, K. Beckert, Fourier versus non-Fourier heat conduction in materials with a nonhomogeneous inner structure, J. Heat Transfer 122 (2000) 363-365.

[13] E. P. Scott, M. Tilahun, B. Vick, The question of thermal waves in heterogeneous and biological materials, J. Biomech. Eng. 131 (2009) 074518 .

[14] C. L. Tien, A. Majumdar, M. I. Flik, S. Kotake, K. Hijikata, Y. Hayashi, Molecular and microscale transport phenomena: A report of the JapanU.S. joint seminar, Ann. Rev. Heat Transfer 6 (1995) 355-377.

[15] M. Asheghi, Microscale heat transfer, http://www.electronics-cooling.com/2007/02/microscale-heat-transfer/. 
[16] M. Asheghi, M. N. Touzelbaev, K. E. Goodson, Y. K. Leung, S. S. Wong, Temperature-dependent thermal conductivity of single-crystal silicon layers in SOI substrates, J. Heat Transfer 120 (1998) 30-36.

[17] D. G. Cahill, W. K. Ford, K. E. Goodson, G. D. Mahan, A. Majumdar, H. J. Maris, R. Merlin, S. R. Philpot, Nanoscale thermal transport, J. Appl. Phys. 93 (2003) 793-818.

[18] N. J. Pilgrim, W. Batty, R. W. Kelsall, C. Snowden, Nanoscale electrothermal co-simulation: compact dynamic models of hyperbolic heat transport and self-consistent device Monte Carlo, Microelectronics Journal 35 (2004) 823-830.

[19] W. B. Lor, H. S. Chu, Hyperbolic heat conduction in thin-film high Tc superconductors with interface thermal resistance, Cryogenics 39 (1999) 739-750.

[20] G. J. Wagner, R. E. Jones, J. A. Templeton, M. L. Parks, An atomisticto-continuum coupling method for heat transfer in solids, Comput. Methods Appl. Mech. Engrg. 197 (2008) 3351-3365.

[21] D. E. Glass, M. N. Özişik, B. Vick, Non-Fourier effects on transient temperature resulting from periodic on-off heat flux, Int. J. Heat Mass Transfer 30 (8) (1987) 1623-1631.

[22] M. Human, Non-Fourier heat transfer in laser heated metal surfaces, in: J. H. K. et al. (Ed.), Heat Transfer: Korea-USA Seminar, 1986, pp. $521-533$.

[23] D. Y. Tzou, The resonance phenomenon in thermal waves, Int. J. Eng. Sci. 29 (1991) 1167-1177.

[24] D. Y. Tzou, Resonance of thermal waves under frequency excitations, Fundamental in Conduction, ASME HTD 173 (1991) 11-27.

[25] D. Y. Tzou, Thermal resonance under frequency excitations, J. Heat Transfer 114 (2) (1992) 310-316.

[26] D. Y. Tzou, Damping and resonance phenomenon of thermal waves, J. Appl. Mech. 59 (4) (1992) 862-867. 
[27] M. Flik, C. L. Tien, Size effects on the thermal conductivities of high-Tc thin-film superconductors, J. Heat Transfer 112 (1990) 872-881.

[28] G. Espinosa-Paredes, E. G. Espinosa-Martinez, Fuel rod model based on non-Fourier heat conduction equation, Annals of Nuclear Energy 36 (2009) 680-693.

[29] P. Galenko, D. Jou, Diffuse-interface model for rapid phase transformations in nonequilibrium systems, Phys. Rev. E 71 (2005) 046125-.

[30] J. Jiang, Convergence to equilibrium for a fully hyperbolic phase-field model with Cattaneo heat flux law, Math. Meth. Appl. Sci. 32 (2009) $1156-1182$.

[31] S. Bargmann, R. Greve, P. Steinmann, Simulation of cryovolcanism on Saturn's moon Enceladus with the Green-Naghdi theory of thermoelasticity, Bulletin of Glaciological Research 26 (2008) 23-32.

[32] L. Herrera, N. Falcón, Heat waves and thermohaline instability in a fluid, Physics Letters A 201 (1995) 33-37.

[33] Y. Dolak, T. Hillen, Cattaneo models for chemosensitive movements, J. Math Biology 46 (2003) 460-478.

[34] J. Liu, Preliminary survey on the mechanisms of the wave-like behaviors of heat transfer in living tissues, Forschung in Ingenieurwesen 66 (2000) $1-10$.

[35] A. B. Duncan, G. P. Peterson, Review of microscale heat transfer, Appl. Mech. Rev. 47 (9) (1994) 397-428.

[36] M. N. Özişik, D. Y. Tzou, On the wave theory in heat conduction, J. Heat Transfer 116 (1994) 526-535.

[37] A. J. C. Ladd, B. Moran, W. G. Hoover, Lattice thermal conductivity: A comparison of molecular dynamics and anharmonic lattice dynamics, Phys. Rev. B 34 (8) (1986) 5058-5064.

[38] P. K. Schelling, S. R. Phillpot, P. Keblinski, Comparison of atomic-level simulation methods for computing thermal conductivity, Phys. Rev. B 65 (14) (2002) 144306. 
[39] D. H. Tsai, R. A. MacDonald, Molecular-dynamical study of second sound in a solid excited by a strong heat pulse, Phys. Rev. B 14 (10) (1976) 4714-4723.

[40] S. Volz, J. B. Saulnier, M. Lallemand, B. Perrin, P. Depondt, M. Mareschal, Transient Fourier-law deviation by molecular dynamics in solid argon, Phys. Rev. B 54 (1) (1996) 340-347.

[41] M. A. Osman, D. Srivastava, Molecular dynamics simulations of heat pulse propagation in single-wall carbon nanotubes, Phys. Rev. B 72 (12) (2005) 125413.

[42] J. Shiomi, S. Maruyama, Non-Fourier heat conduction in a single-walled carbon nanotube: Classical molecular dynamics simulations, Phys. Rev. B 73 (20) (2006) 205420.

[43] Q. Liu, P. Jiang, H. Xiang, Molecular dynamics simulations of nonFourier heat conduction, Prog. Nat. Sci. 18 (2008) 999-1007.

[44] S. P. A. Gill, Nonequilibrium molecular dynamics and multiscale modeling of heat conduction in solids, in: T. Dumitrica (Ed.), Trends in Computational Nanomechanics, Vol. 9, Springer, 2010, pp. 85-134.

[45] A. Tenenbaum, G. Ciccotti, R. Gallico, Stationary nonequilibrium states by molecular dynamics, Phys. Rev. A 25 (5) (1982) 27778-2787.

[46] K. Aoki, D. Kusnezov, Bulk properties of anharmonic chains in strong thermal gradients: non-equilibrium phi4 theory, Phys. Lett. A 265 (4) (2000) 250-256.

[47] L. P. Pitaevskii, E. M. Lifshitz, Physical Kinetics, ButterworthHeinenann, Oxford, UK, 1995.

[48] D. A. Young, H. J. Maris, Lattice-dynamical calculations of the Kapitza resistance between fcc lattices, Phys. Rev. B 40 (1989) 3685-3693.

[49] A. V. Savin, O. V. Gendelman, Heat conduction in one-dimensional lattices with on-site potential, Phys. Rev. E 67 (4) (2003) 041205-1041205-12.

[50] S. Lepri, R. Livi, A. Politi, Thermal conduction in classical lowdimensional lattices, Physics Reports 377 (2003) 1-80. 
[51] K. Jolley, S. P. A. Gill, Modelling transient heat conduction in solids at multiple length and time scales: a coupled non-equilibrium MDcontinuum approach, J. Comput. Phys. 228 (19) (2009) 7412-7425.

[52] A. Singh, E. B. Tadmor, Removing artificial kapitza effects from bulk thermal conductivity calculations in direct molecular dynamics, J. Appl. Phys. 117 (2015) 185101.

[53] B. C. Daly, H. J. Maris, K. Imamura, S. Tamura, Molecular dynamics calculation of the thermal conductivity of superlattices, Phys. Rev. B 66 (2002) 024301.

[54] H. Poincaré, Théorie analytique de la propagation de la chaleur, University of Michigan Library, 1895.

[55] M. E. Gurtin, A. C. Pipkin, A general theory of heat conduction with finite wave speeds, Arch. Rational Mech. Anal. 31 (22) (1968) 113-126.

[56] J. W. Nunziato, On heat conduction in materials with memory, Quart. Appl. Math. 29 (2) (1971) 187-204.

[57] H. Jeffreys, The Earth: Its Origin, History and Physical Constitution, 8th Edition, Cambridge University Press, Cambridge, 1976.

[58] D. D. Joseph, A. Narain, O. Riccius, Shear-wave speeds and elastic moduli for different liquids. Part 1. Theory, J. Fluid Mech. 171 (1986) 289-308.

[59] J. C. Maxwell, On the dynamical theory of gases, Philos. Trans. Royal Soc. London 157 (1867) 49-88.

[60] H. Markovitz, Boltzmann and the beginnings of linear viscoelasticity, Trans. Soc. Rheol. 21 (3) (1977) 381-388.

[61] D. Y. Tzou, An engineering assessment to the relaxation-time in thermal wave-propagation, Int. J. Heat Mass Transfer 36 (7) (1993) 1845-1851.

[62] D. Y. Tzou, A unified field approach for heat conduction from macroto micro-scales, J. Heat Transfer 117 (1) (1995) 8-16.

[63] J. Jellinek, D. H. Li, Separation of the energy of overall rotation in any N-body system, Phys. Rev. Lett. 62 (3) (1989) 241-244. 
[64] E. B. Tadmor, R. E. Miller, Modeling Materials, Cambridge University Press, Cambridge, 2011.

[65] D. J. Evans, G. Morriss, Statistical Mechanics of Nonequilibrium Liquids, 2nd Edition, Cambridge University Press, New York, US, 2008.

[66] J. H. Irving, J. G. Kirkwood, The statistical mechanical theory of transport processes. IV. The equations of hydrodynamics, J. Chem. Phys. 18 (6) (1950) 817-829.

[67] S. G. Volz, G. Chen, Molecular dynamics simulation of thermal conductivity of silicon nanowire, Appl. Phys. Lett. 75 (14) (1999) 2056-2058.

[68] N. C. Admal, E. B. Tadmor, Stress and heat flux for arbitrary multibody potentials: A unified framework, J. Chem. Phys. 134 (2011) 184106.

[69] X. W. Zhou, S. Aubry, R. E. Jones, A. Greenstein, P. K. Schelling, Towards more accurate molecular dynamics calculation of thermal conductivity: Case study of GaN bulk crystals, Phys. Rev. B 79 (11) (2009) 115201-1-115201-17.

[70] D. P. Sellan, E. S. Landry, J. E. Turney, A. J. H. McGaughey, C. H. Amon, Size effects in molecular dynamics thermal conductivity predictions, Phys. Rev. B 81 (2010) 214305.

[71] J. E. Jones, On the determination of molecular fields. I. From the variation of the viscosity of a gas with temperature, Proc. Roy. Soc. A 106 (1924) 441-462.

[72] J. E. Jones, On the determination of molecular fields. I. From the equation of state of a gas, Proc. Roy. Soc. A 106 (1924) 463-477.

[73] T. X. Nguyen, S. K. Bhatia, D. Nicholson, Prediction of high-pressure adsorption equilibrium of supercritical gases using density functional theory, Langmuir 21 (7) (2005) 3187-3197.

[74] E. B. Tadmor, Truncated lennard-jones model for Ar with parameters from Nguyen, https://openkim.org/cite/MO_398194508715_000.

[75] E. B. Tadmor, Driver for the Lennard-Jones model truncated to have zero energy above the cutoff radius, https://openkim.org/cite/MD_132729421025_000. 
[76] E. B. Tadmor, R. S. Elliott, J. P. Sethna, R. E. Miller, C. A. Becker, The potential of atomistic simulations and the Knowledgebase of Interatomic Models, JOM 63 (2011) 17.

[77] S. Nosé, A unified formulation of the constant temperature molecular dynamics methods, J. Chem. Phys. 81 (1) (1984) 511-519.

[78] S. Nosé, A molecular dynamics method for simulations in the canonical ensemble, Mol. Phys. 52 (2) (1984) 255-268.

[79] W. G. Hoover, Canonical dynamics: Equilibrium phase-space distributions, Phys. Rev. A 31 (3) (1985) 1695-1697.

[80] W. G. Hoover, Constant pressure equations of motion, Phys. Rev. A 34 (3) (1986) 2499-2500.

[81] G. J. Martyna, M. L. Klein, M. Tuckerman, Nosé-hoover chains: The canonical ensemble via continuous dynamics, J. Chem. Phys. 97 (4) (1992) 2635-2645.

[82] D. Frenkel, B. Smit, Understanding Molecular Simulation, 2nd Edition, Academic Press, San Diego, CA, US, 2002.

[83] T. Schneider, E. Stoll, Molecular-dynamics study of a three-dimensional one-component model for distortive phase transitions, Phys. Rev. B 17 (3) (1978) 1302-1322.

[84] S. J. Plimpton, Fast parallel algorithms for short-range molecular dynamics, J. Comput. Phys. 117 (1995) 1-19.

[85] LAMMPS website, http://lammps.sandia.gov/.

[86] W. Shinoda, M. Shiga, M. Mikami, Rapid estimation of elastic constants by molecular dynamics simulation under constant stress, Phys. Rev. B 69 (13) (2004) 134103-1-134103-8.

[87] D. J. Tobias, G. J. Martyna, M. L. Klein, Molecular dynamics simulations of a protein in the canonical ensemble, J. Phys. Chem. 97 (49) (1993) 12959-12966.

[88] M. Parrinello, A. Rahman, Crystal structure and pair potentials: A molecular-dynamics study, Phys. Rev. Lett. 45 (14) (1980) 1196-1199. 
[89] G. J. Keeler, D. N. Batchelder, Measurement of the elastic constants of argon from 3 to 77 degrees K, J. Phys. C: Solid State Phys. 3 (1970) $510-522$.

[90] M. P. Allen, D. J. Tildesley, Computer Simulation of Liquids, Oxford University Press, New York, 1987.

[91] H. Kaburaki, J. Li, S. Yip, H. Kimizuka, Dynamical thermal conductivity of argon crystal, J. Appl. Phys. 102 (4) (2007) 043514-1-043514-6.

[92] R. A. Guyer, J. A. Krumhansl, Solution of the linearized Boltzmann equation, Phys. Rev. 148 (2) (1966) 766-778.

[93] R. Berman, Thermal Conduction in Solids, Oxford University Press, Oxford, 1976.

[94] H. E. Jackson, C. T. Walker, Thermal conductivity, second sound, and phonon-phonon interactions in NaF, Phys. Rev. B 3 (4) (1971) 14281439 .

[95] C. Feldman, M. L. Klein, On the velocity of sound in solid argon, Phys. Lett. A 25 (3) (1967) 190-191.

[96] L. Finegold, N. E. Phillips, Low-temperature heat capacities of solid argon and krypton, Phys. Rev. 177 (1969) 1383-1391. 